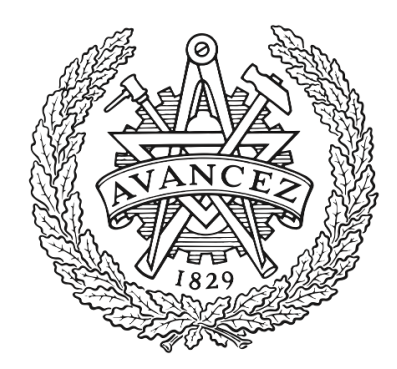

\title{
CHALMERS
}

UNIVERSITY OF TECHNOLOGY

\section{A direct numerical simulation study of the influence of flame-generated vorticity on reaction-zone-surface area in weakly turbulent premixed}

Downloaded from: https://research.chalmers.se, 2023-04-26 11:28 UTC

Citation for the original published paper (version of record):

Lipatnikov, A., Sabelnikov, V., Nishiki, S. et al (2019). A direct numerical simulation study of the influence of flame-generated vorticity on reaction-zone-surface area in weakly turbulent premixed combustion. Physics of Fluids, 31(5). http://dx.doi.org/10.1063/1.5094976

N.B. When citing this work, cite the original published paper. 
- A Direct Numerical Simulation Study of the Influence of - Flame-Generated Vorticity on Reaction-Zone-Surface 3 Area in Weakly Turbulent Premixed Combustion

A.N. Lipatnikov, ${ }^{1, \text { a) }}$ V.A. Sabelnikov, ${ }^{2,3}$ S. Nishiki, ${ }^{4}$ and T. Hasegawa ${ }^{5}$

${ }^{1)}$ Department of Mechanics and Maritime Sciences, Chalmers University of Technology, Gothenburg, 412 96, Sweden

${ }^{2)}$ ONERA - The French Aerospace Lab., F-91761 Palaiseau,

${ }^{3)}$ Central Aerohydrodynamic Institute (TsAGI), 140180 Zhukovsky, Moscow Region, Russian Federation

4) Department of Mechanical Engineering, Kagoshima University, Kagoshima 890-0065, Japan

5) Institute of Materials and Systems for Sustainability, Nagoya University, Nagoya 464-8603, Japan

(Dated: 8 April 2019)

Direct numerical simulation data obtained from two statistically stationary, onedimensional, planar, weakly turbulent, premixed flames are analyzed in order to examine the influence of flame-generated vorticity on the surface area of the reaction zone. The two flames are associated with the flamelet combustion regime and are characterized by two significantly different density ratios $\sigma=7.53$ and 2.5 , with all other things being roughly equal. The obtained results indicate that generation of vorticity due to baroclinic torque within flamelets can impede wrinkling the reaction surface, reduce its area, and, hence, decrease the burning rate. Thus, these results call for revisiting the widely-accepted concept of combustion acceleration due to flame-generated turbulence. In particular, in the case of $\sigma=7.53$, the local stretch rate, which quantifies the local rate of an increase or decrease in the surface area, is predominantly negative in regions characterized by a large magnitude of enstrophy or a large magnitude of the baroclinic torque term in the enstrophy transport equation, with the effect being more pronounced at larger values of the mean combustion progress variable. If the density ratio is low, e.g., $\sigma=2.5$, the baroclinic torque weakly effects the vorticity field within the mean flame brush and the aforementioned effect is not pronounced.

PACS numbers: 47.70.Fw, 82.33.Vx, 47.27.-i

Keywords: premixed turbulent burning, flame-generated turbulence, thermal expansion, combustion acceleration, DNS

\section{INTRODUCTION}

Interaction of a turbulent flow and an exothermic reaction wave is a highly non-linear and multiscale phenomenon relevant to various processes ranging from combustion ${ }^{1-9}$ and deflagration-todetonation transition ${ }^{10}$ under terrestrial conditions to evolution of thermonuclear Ia supernovae ${ }^{11,12}$ in the Universe. While the governing physical mechanisms of the influence of turbulence on a reaction wave are sufficiently well understood, ${ }^{13-19}$ the problem of the influence of thermal expansion in the wave on the incoming turbulent flow still strongly challenges the research community.

For instance, almost seven decades ago, Karlovitz et al. ${ }^{20}$ and Scurlock and Grover ${ }^{21}$ put forward a seminal concept of combustion acceleration due to flame-generated turbulence in order to explain unexpectedly high burning rates obtained in some early experiments. For that purpose, they (i) highlighted two different (in the two different papers cited above) physical mechanisms of turbulence 
generation due to combustion-induced thermal expansion and (ii) hypothesized that such a flamegenerated turbulence significantly increased the flame speed $S_{T}$, with the influence of the flamegenerated turbulence on $S_{T}$ being assumed to be basically similar to the influence of the incoming turbulence on $S_{T}$. Since that pioneering studies, the flame-generated turbulence and other thermal expansion effects were in the focus of research into premixed turbulent combustion, but progress in understanding and modeling them has yet been rather moderate, as reviewed elsewhere. ${ }^{22-25} \mathrm{Nev}$ ertheless, to the best of the present authors' knowledge, the classical concept ${ }^{20,21}$ of -combustion acceleration due to flame-generated turbulence has never been disputed, at least in the case of weak or moderate turbulence associated with a well-pronounced increase ${ }^{26}$ in $S_{T}$ by the rms turbulent velocity $u^{\prime}$.

On the one hand, this concept is indirectly supported by well-documented self-acceleration of large-scale laminar flames, ${ }^{27-33}$ which is commonly attributed to development of the flame instabilities, ${ }^{29-33}$ followed by generation of turbulence due to combustion-induced thermal expansion. ${ }^{27,28,34}$

On the other hand, certain fundamental issues associated with that concept have not yet been resolved properly. In particular, first, while the physical mechanisms highlighted by Karlovitz et $\mathrm{al}^{20}$ and by Scurlock and Grover ${ }^{21}$ are relevant to turbulence downstream of the instantaneous flame, the influence of a premixed flame on the turbulent flow upstream of the flame has yet been understood poorly. However, since the flame propagates into the unburned gas, perturbations of the incoming turbulent flow are required in order for the thermal expansion effects to cause selfacceleration of the flame.

Second, in the constant-density flow of unburned reactants, combustion-induced flow perturbations can differ fundamentally from the incoming turbulence. For instance, while the rotational motion dominates in a typical constant-density turbulent flow, the unburned-reactant-flow perturbations that are directly caused by the flame-generated pressure perturbations are expected to be irrotational, because the sole term that involves the pressure gradient in the transport equation ${ }^{35}$ for vorticity (i.e., the baroclinic torque term) vanishes in the constant-density flow of the unburned reactants. Indeed, certain DNS data indicate that the irrotational velocity component is increased (when compared to the rotational component) upstream and in the vicinity of a premixed flame in a weakly turbulent flow. ${ }^{25}$

Third, within a flame, rotational flow perturbations generated due to thermal expansion effects, e.g., vorticity generation due to baroclinic torque, ${ }^{17,24}$ and the incoming turbulent eddies can affect the flame surface area and, hence, the burning rate in opposite directions, i.e., the former rotational perturbations can mitigate an increase in the area under the influence of the incoming turbulence. To the best of the present authors' knowledge, such a scenario was not discussed in the turbulent combustion literature until recently. ${ }^{36}$ On the contrary, the influence of the flame-generated turbulence on $S_{T}$ is typically assumed to be basically equivalent to the influence of the incoming turbulence on $S_{T}$, i.e., both kinds of turbulence are often considered to increase $S_{T}$ in a similar manner. Nevertheless, there are theoretical and qualitative reasons for hypothesizing the former, commonly disregarded scenario, i.e., the reduction of flame-surface area, caused by the rotational motion induced due to thermal expansion in the flame.

Indeed, first, the well-recognized theory of the hydrodynamic instability of a laminar premixed flame ${ }^{37}-39$ addresses an infinitely thin flame front in a $2 \mathrm{D}$ irrotational flow of unburned reactants and predicts generation of vorticity in the combustion products just downstream of the front if its shape is weakly perturbed. However, inspection of the relevant theoretical expressions, e.g., see Eq. (40) in a review paper, ${ }^{24}$ shows that the rotational component of the product velocity works to smooth out perturbations of the front shape, i.e., to mitigate the instability. Nevertheless, the hydrodynamic instability develops under the influence of the irrotational flow perturbations, which overwhelm the rotational ones (at the same time, it is worth remembering that the vorticity generation behind the front is the direct consequence of the momentum conservation at the front, i.e., if the flow around the flame is described by the continuity and Euler or Navier-Stokes equations, then, the flame instability is always accompanied by the generation of vorticity in the burned products). We may also note that, by theoretically studying an irrotational model of the hydrodynamic instability, put forward by Frankel $^{40}$ (within the framework of this model, momentum is not conserved ${ }^{41}$ at the flame front), Sivashinsky and Clavin ${ }^{41}$ have found that "neglect of vorticity generation increases the perturbation growth rate." 
(a) convex reaction surface

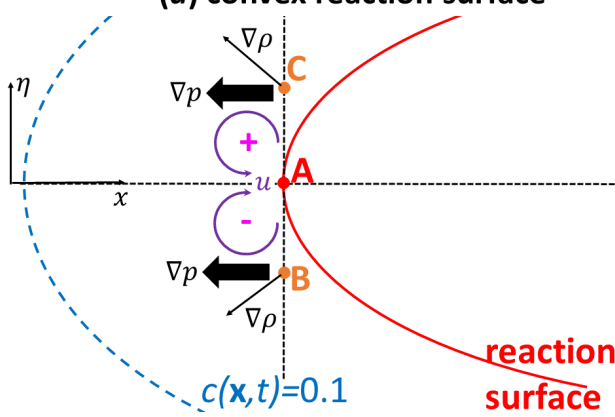

(b) concave reaction surface

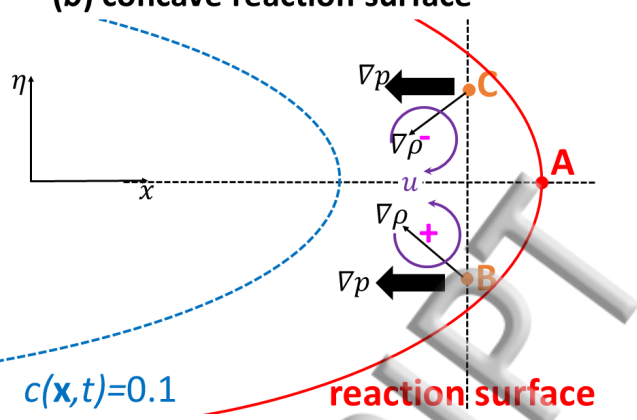

FIG. 1. Generation of vorticity by baroclinic torque within flamelets in the vicinity of reaction zones (a) convex or (b) concave towards unburned gas.

Second, to illustrate that the flame-generated vorticity can impede growing the flame surface area and the burning rate in a turbulent flow, let us consider flow within a flamelet preheat zone in the vicinity of a reaction zone convex or concave towards the unburned gas, see Figs. 1a and $1 \mathrm{~b}$, respectively. Here, the cold boundary of the preheat zone and the reaction surface are shown in blue dashed and red solid lines, respectively. The turbulent flame is statistically 1D, planar, normal to the $x$-axis, and propagates from right to left.

Let us consider the well-known vorticity transport equation ${ }^{24,35}$

$$
\frac{\partial \omega_{i}}{\partial t}+u_{k} \frac{\partial \omega_{i}}{\partial x_{k}}=\underbrace{\omega_{k} \frac{\partial u_{i}}{\partial x_{k}}}_{\boldsymbol{T}_{1}}+\underbrace{\varepsilon_{i j k} \frac{\partial}{\partial x_{j}}\left(\frac{1}{\rho} \frac{\partial \tau_{k l}}{\partial x_{l}}\right)}_{\boldsymbol{T}_{2}} \underbrace{-\omega_{i} \frac{\partial u_{k}}{\partial x_{k}}}_{\boldsymbol{T}_{3}}+\underbrace{\frac{1}{\rho^{2}} \varepsilon_{i j k} \frac{\partial \rho}{\partial x_{j}} \frac{\partial p}{\partial x_{k}}}_{\boldsymbol{T}_{4}},
$$

where $t$ is the time, $x_{i}$ are spatial coordinates, $\omega_{i}$ and $u_{i}$ are components of the vorticity $\boldsymbol{\omega}=\nabla \times \boldsymbol{u}$ and velocity $\boldsymbol{u}$ vectors, respectively, $\rho$ is the density, $p$ is the pressure,

$$
\tau_{i j}=\rho v\left(\frac{\partial u_{i}}{\partial x_{j}}+\frac{\partial u_{j}}{\partial x_{i}}-\frac{2}{3} \delta_{i j} \frac{\partial u_{k}}{\partial x_{k}}\right)
$$

is the viscous stress tensor, $\delta_{i j}$ is the Kronecker delta, $\varepsilon_{i j k}$ is the cyclic permutation tensor, and the summation convention applies for the repeated indexes $k$ and $l$.

The focus of the present study is placed on the baroclinic torque term $\boldsymbol{T}_{4}$ or $\boldsymbol{B}_{\boldsymbol{\omega}}=(\nabla \rho \times \nabla p) / \rho^{2}$ and on the counterpart term $B_{\omega} \cdot \omega=\omega \cdot B_{\omega}$ in the following transport equation ${ }^{42}$

$$
\frac{\partial \omega^{2}}{\partial t}+u_{k} \frac{\partial \omega^{2}}{\partial x_{k}}=\underbrace{\omega_{i} \omega_{k} \frac{\partial u_{i}}{\partial x_{k}}}_{T_{1}}+\underbrace{\varepsilon_{i j k} \omega_{i} \frac{\partial}{\partial x_{j}}\left(\frac{1}{\rho} \frac{\partial \tau_{k l}}{\partial x_{l}}\right)}_{T_{2}} \underbrace{-2 \omega^{2} \frac{\partial u_{k}}{\partial x_{k}}}_{T_{3}}+\underbrace{\frac{\varepsilon_{i j k} \omega_{i}}{\rho^{2}} \frac{\partial \rho}{\partial x_{j}} \frac{\partial p}{\partial x_{k}}}_{T_{4}}
$$

for enstrophy $\omega^{2}=\omega \cdot \omega / 2$, because these two terms directly involve the density gradient and control $^{42}$ vorticity generation in weakly turbulent flames characterized by a large Bray ${ }^{23}$ number $N_{B} \propto(\sigma-1) S_{L} / u^{\prime}$, in particular, in flame $\mathrm{H}$ analyzed in Section III, see figure 9b in Ref. [42]. Here, $S_{L}$ is the laminar flame speed, $\sigma=\rho_{u} / \rho_{b}$ is the density ratio, subscripts $u$ and $b$ designate unburned reactants and burned products, respectively. For the goals of the present study, the weakly turbulent flames are of paramount interest, because the influence of combustion-induced thermal expansion on the incoming turbulence is most pronounced under such conditions ${ }^{24,25}$, whereas turbulence is weakly affected by thermal expansion in highly turbulent flames ${ }^{43,44}$. For instance, at $N_{B}<$ 1 , vorticity generation is mainly controlled ${ }^{1,45,46}$ by the vortex-stretching terms $T_{1}$ and $T_{1}$, which involve neither density nor viscosity, but are indirectly affected by combustion-induced thermal expansion, which changes the velocity field.

It is also worth noting that, first, the dilatation terms $\boldsymbol{T}_{3}$ and $T_{3}$ always reduce the incoming vorticity. Second, while the viscous term $\boldsymbol{T}_{2}$ can re-distribute vorticity within weakly turbulent flames ${ }^{42}$, the mean viscous term $T_{2}$ reduces the mean enstrophy ${ }^{1,42,45,46}$ both at large and small $N_{B}$.

Let us consider behaviour of a transverse $\left(\eta=y\right.$ or $z$ ) component of the vector $\boldsymbol{B}_{\boldsymbol{\omega}}$, as well as behaviour of the local pressure and density gradients. Note that the two gradients are not parallel to each other within a turbulent flame, because the local pressure is affected by the surrounding 
This manuscript was accepted by Phys. Fluids. Click here to see the version of record.

velocity field. For instance, if the Mach number asymptotically vanishes, the local pressure is determined by the entire velocity field according to Poisson equation, ${ }^{35}$ i.e. pressure perturbations propagate at an infinitely high speed in this limiting case. Even under laminar-flow conditions, the Darrieus-Landau solution ${ }^{37}$ shows that $\nabla p$ is not normal to the instantaneous flame front, see Eq. (40) in a review paper. ${ }^{24}$

Accordingly, to simplify the following qualitative discussion, let us assume that the local pressure gradient is parallel to the $x$-axis. Indeed, in a typical premixed turbulent flame, there is always a significant axial pressure gradient due to the mean pressure drop from the leading to the trailing edge of the mean flame brush, but small transverse pressure gradients $\left|\nabla_{y} p\right|$ and $\left|\nabla_{z} p\right|$ can also play a role, as discussed in Section III.A. Then, the mutual orientation of $\nabla p$ (bold black arrows) and the projection of the vector $\nabla \rho$ (see fine black arrows) on the transverse plane shown in Fig. 1a indicates that the normal (to the plane) component of the vector $\boldsymbol{B}_{\boldsymbol{\omega}}$ points to (from) the reader at positive (negative) values of the local transverse coordinate $\eta$ counted from the transverse coordinate of the locally leading point $\mathrm{A}$, see red circle. More specifically, $B_{\boldsymbol{\omega}, y}<0$ if the local $\eta=z-z_{A}>0$, but $B_{\boldsymbol{\omega}, y}>0$ if the $\eta=z-z_{A}<0$, whereas $B_{\boldsymbol{\omega}, z}>0$ if the $\eta=y-y_{A}>0$, but $B_{\boldsymbol{\omega}, z}<0$ if the $\eta=y-y_{A}<0$. Here, $y_{A}$ and $z_{A}$ are the $y$ and $z$-coordinates of the locally leading point A.

Accordingly, baroclinic torque locally works to generate a vortex pair, see violet arcs, with its "symmetry" axis being parallel to the $x$-axis, see horizontal dashed straight line. Moreover, by virtue of the aforementioned orientation of the normal (to the figure plane) component of $\boldsymbol{B}_{\boldsymbol{\omega}}$, the axial velocity component $u$ associated with such a vortex pair is positive in the vicinity of the symmetry axis, see violet arrows. Therefore, the local axial velocity associated with the local flame-generated vorticity pushes the leading point inside the mean flame brush, thus, reducing the reaction-surface area and, consequently, the turbulent burning rate.

A similar conclusion regarding reduction of the reaction-surface area due to the vorticity generated by baroclinic torque can be drawn by considering Fig. $1 \mathrm{~b}$, where the behaviors of $\nabla \rho, \nabla p, \boldsymbol{B}_{\boldsymbol{\omega}}$, $\boldsymbol{\omega}$, and $\boldsymbol{u}$ are sketched in the vicinity of a locally trailing point $A$ on the reaction surface concave to the unburned reactants.

Thus, both the theory of the hydrodynamic instability of laminar premixed flames and the physical scenarios sketched in Fig. 1 imply that, contrary to the widely accepted concept of combustion acceleration due to flame-generated turbulence, ${ }^{20,21}$ the flame-generated vorticity can impede growing the reaction-surface area, thus, reducing the turbulent burning rate. The major goal of the present work is to examine this hypothesis, which has yet been beyond the focus of the mainstream research into flame-turbulence interaction.

To fill this gap, Direct Numerical Simulation (DNS) data generated by two of the present authors ${ }^{47,48}$ more than 15 years ago were analyzed. The first results of this analysis were briefly reported in a recent letter ${ }^{36}$ but the present paper significantly extends discussion and assessment of the aforementioned hypothesis.

The choice of this DNS database, which may appear to be outdated when compared to recent DNS data ${ }^{49-56}$ generated in the case of complex combustion chemistry and a high ratio of the rms turbulent velocity $u^{\prime}$ to the laminar flame speed $S_{L}$, requires comments. Since the focus of the following discussion is placed on the influence of combustion-induced thermal expansion on the velocity, pressure, vorticity, and enstrophy fields upstream of reaction zones, detailed description of complex combustion chemistry within such zones appears to be of secondary importance when compared to two other major requirements. First, in order to make the studied thermal expansion effects as strong as possible, the heat release and density drop should be localized to sufficiently thin zones and the velocity jumps across such zones should be sufficiently large when compared to the rms turbulent velocity $u^{\prime}$. In other words, the flamelet regime ${ }^{13-15,18,57}$ of premixed turbulent combustion associated with $u^{\prime} / S_{L}=\mathrm{O}(1)$ and $N_{B}>1$ should be addressed. The selected DNS data are indeed associated with this regime, as discussed in detail elsewhere, ${ }^{58}$ whereas the vast majority of recent very advanced DNS studies attacked other combustion regimes characterized by a large $u^{\prime} / S_{L}$.

Second, to better explore the thermal expansion effects, data obtained at significantly different density ratios $\sigma=\rho_{u} / \rho_{b}$ are required. The selected DNS database does satisfy this requirement, because cases of $\sigma=2.5$ and 7.53 were simulated, with all other things being roughly equal. As discussed in detail elsewhere, ${ }^{42}$ such variations in the density ratio offer an opportunity to explore 
two opposite scenarios, which are directly relevant to the major goal of the present study. These are; (i) the generation of vorticity due to baroclinic torque overwhelms the dissipation of vorticity due to dilatation and viscous forces, thus, increasing enstrophy within the flame brush at $\sigma=7.53$, but (ii) the dilatation and dissipation effects dominate and reduce the enstrophy at $\sigma=2.5$. Therefore, the selected DNS data appear to be fully adequate to the major goal of the present work.

The paper is organized as follows. In the next section, the DNS attributes are reported. Simulated results are discussed in Section III, followed by conclusions.

\section{DIRECT NUMERICAL SIMULATIONS}

Since the DNS data were discussed in detail elsewhere 47,48 and were already used by various research groups, ${ }^{42,58-75}$ let us restrict ourselves to a very brief summary of those compressible 3D simulations. They dealt with statistically 1D and planar, equidiffusive, adiabatic flames modeled by unsteady continuity, Navier-Stokes, and energy equations, supplemented with the ideal gas state equation and a transport equation for the mass fraction $Y$ of a deficient reactant. Temperaturedependence of molecular transport coefficients was taken into account, e.g., the kinematic viscosity $v=v_{u}\left(T / T_{u}\right)^{0.7}$, where $T$ is the temperature. The Lewis $L e$ and Prandtl $\operatorname{Pr}$ numbers were equal to 1.0 and 0.7 , respectively. Combustion chemistry was reduced to a single reaction. Therefore, the mixture state was characterized with a single combustion progress variable $c=\left(T-T_{u}\right) /\left(T_{b}-T_{u}\right)=$ $1-Y / Y_{u}$.

The computational domain was a rectangular box $\Lambda_{x} \times \Lambda_{y} \times \Lambda_{z}$, where $\Lambda_{x}=8 \mathrm{~mm}$ and $\Lambda_{y}=$ $\Lambda_{z}=4 \mathrm{~mm}$. It was resolved using a uniform rectangular $(2 \Delta x=\Delta y=\Delta z)$ mesh of $512 \times 128 \times 128$ points. The flow was periodic in $y$ and $z$ directions.

Using an energy spectrum $E(\kappa)$ proposed by Kraichnan ${ }^{76}$ and setting $u_{0}^{\prime}=0.53 \mathrm{~m} / \mathrm{s}$ and an integral length scale

$$
L=\frac{3 \pi}{4} \frac{\int_{0}^{\infty} \kappa^{-1} E(\kappa) d \kappa}{\int_{0}^{\infty} E(\kappa) d \kappa}
$$

equal to $3.45 \mathrm{~mm}$, homogeneous isotropic turbulence was generated ${ }^{47}$ in a separate box and was injected into the computational domain through the left boundary $x=0$. While the used value of the length scale $L$ was comparable with the width $\Lambda_{y}=\Lambda_{z}$ of the computational domain, the velocity fields simulated at $y=\Lambda_{y} / 2$ or $z=\Lambda_{z} / 2$ did not correlate with the velocity fields simulated at the transverse boundaries. For instance, the second-order structure functions of the velocity field, reported recently, ${ }^{73,75}$ level off at transverse distances less than $\Lambda_{y} / 2$ or $\Lambda_{z} / 2$.

At $t=0$, a planar laminar flame was embedded into statistically the same turbulence assigned for the velocity field in the entire computational domain. Subsequently, the mean inflow velocity $U$ was increased twice, i.e., $U\left(0 \leq t<t_{1}\right)=S_{L}<U\left(t_{1} \leq t<t_{2}\right)<U\left(t_{2} \leq t\right)$. In order to keep the flame in the computational domain till the end $t_{3}$ of the simulations, $U\left(t_{2} \leq t\right)$ was close to the mean turbulent flame speed $\overline{S_{T}}$ averaged over a time interval of $\left(t_{2}, t_{3}\right)$.

Three DNS data sets H, M, and L associated with High, Medium, and Low, respectively, density ratios $\sigma$ were originally generated. ${ }^{47,48}$ Since the focus of the present study is placed on thermal expansion effects, the following discussion will be restricted to results obtained in two cases characterized by the highest and the lowest density ratios, i.e., flame $\mathrm{H}\left(\sigma=7.53, S_{L}=0.6 \mathrm{~m} / \mathrm{s}\right.$, $\left.\delta_{L}=0.217 \mathrm{~mm}\right)$ and flame $\mathrm{L}\left(\sigma=2.5, S_{L}=0.416 \mathrm{~m} / \mathrm{s}, \delta_{L}=0.158 \mathrm{~mm}\right)$. In both cases, $\overline{S_{T}} / S_{L}=1.9$. Here, $\delta_{L}=\left(T_{b}-T_{u}\right) / \max \{|\nabla T|\}$ is the laminar flame thickness. The two flames are well associated with the flamelet combustion regime, e.g., various Bray-Moss-Libby (BML) expressions hold in cases $\mathrm{H}$ and $\mathrm{L}$, see figures 1-4 in an earlier paper. ${ }^{58}$ Since the turbulence decays along the direction $x$ of the mean flow, the turbulence characteristics are slightly different at the leading edges of the $\mathrm{H}$ and L-flame brushes, e.g., $u^{\prime}=0.33 \mathrm{~m} / \mathrm{s}, \lambda=0.43 \mathrm{~mm}, \eta=0.075 \mathrm{~mm}, D a=17.5$, $K a=0.06$ in case $\mathrm{H}$ and $u^{\prime}=0.38 \mathrm{~m} / \mathrm{s}, \lambda=0.47 \mathrm{~mm}, \eta=0.084 \mathrm{~mm}, D a=10.0, K a=0.10$ in case L. Here, $D a=\tau_{T} / \tau_{c}$ and $K a=\tau_{c} u^{\prime} / \lambda$ are the Damköhler and Karlovitz numbers, respectively, $\tau_{c}=v /\left(\operatorname{Pr} S_{L}^{2}\right)$ and $\tau_{T}=\bar{k}^{3 / 2} /\left(u^{\prime} \bar{\varepsilon}\right)$ are the flame and turbulence time scales, respectively, $\lambda=u^{\prime} \sqrt{15 v / \bar{\varepsilon}}$ and $\eta=\left(v^{3} / \bar{\varepsilon}\right)^{1 / 4}$ are the Taylor and Kolmogorov length scales, respectively, $k=\left(u_{k} u_{k}-\bar{u}_{k} \bar{u}_{k}\right) / 2$ and $\varepsilon=2 v S_{i j} S_{i j}$ are the turbulent kinetic energy and its dissipation rate, respectively, $S_{i j}=0.5\left(\partial u_{i} / \partial x_{j}+\partial u_{j} / \partial x_{i}\right)$ is the rate-of-strain tensor, the rms turbulent velocity $u^{\prime}$ is 


\section{Publishing}

equal to $\sqrt{2 \bar{k} / 3}$, and the summation convention applies for repeated indexes.

The DNS data were processed as follows. Mean quantities $\bar{q}=\bar{q}(x)$ were averaged over a transverse plane of $x=$ const and over time ( 221 and 200 snapshots in cases $\mathrm{H}$ and $\mathrm{L}$, respectively, stored during a time interval of $t_{3}-t_{2} \approx 1.5 \mathrm{~L} / u_{0}^{\prime} \approx 10 \mathrm{~ms}$ ). Subsequently, $x$-dependencies were mapped to $\bar{c}$-dependencies using the monotonic spatial profiles of the Reynolds-averaged combustion progress variable $\bar{c}(x)$.

\section{RESULTS AND DISCUSSION}

In this section, two types of numerical results are considered. Data extracted from the entire flame brush are reported in Section III.B. However, before discussing such data, it is worth examining relevance of the qualitative scenarios, sketched in Fig. 1 and discussed in Section I, to premixed turbulent combustion.

Since these scenarios deal with a single point each, they should be assessed by exploring the local velocity, pressure, and density fields in the vicinity of some representative points, with selection of such points being sufficiently arbitrary. In the next subsection, the so-called leading and trailing points are chosen for this purpose. This choice is based on the following three reasons. First, the leading (trailing) points are always convex (concave) towards the unburned gas. Second, since a fluid particle that comes to a leading (trailing) point has been subject to thermal expansion effects during a shorter (longer) travel time counted from some reference point upstream of the flame brush, the effect manifestations are expected to be weakest (strongest) in the vicinity of the leading (trailing) points. Third and the most important, there are physical, ${ }^{16,19,77,78}$ mathematical, ${ }^{79,80}$ and numerical ${ }^{81,82}$ arguments that imply that the leading points play the crucial role in turbulent flame propagation, with such a hypothesis being indirectly supported by recent experimental data. ${ }^{83-85}$ Accordingly, even if the hypothesis still requires further study, an investigation of the local processes in the vicinity of the leading points appears to be of great fundamental interest.

\section{III.A. Leading and trailing points}

In order to examine the qualitative scenarios sketched in Fig. 1, behaviors of vectors $\nabla \rho, \nabla p$, $\boldsymbol{u}, \boldsymbol{\omega}, \boldsymbol{B}_{\boldsymbol{\omega}}$ and scalar quantities such as enstrophy $\omega^{2}$ and baroclinic torque term $B_{\boldsymbol{\omega}} \cdot \boldsymbol{\omega}=\boldsymbol{\omega} \cdot \boldsymbol{B}_{\boldsymbol{\omega}}$ in the transport equation for $\omega^{2}$, were investigated in the vicinity of the leading $x_{l p}(t)$ and trailing $\boldsymbol{x}_{t p}(t)$ points associated with an iso-surface of $c(\boldsymbol{x}, t)=c^{*}$. At each instant $t$, such points were found using the following two constraints applied consecutively. First, the leading $x=x_{l p}(t)$ and trailing $x=x_{t p}(t)$ planes were found using constraints of $c(\boldsymbol{x}, t)<c^{*}$ if $x<x_{l p}(t)$, but $c(\boldsymbol{x}, t) \geq c^{*}$ somewhere on the plane of $x=x_{l p}(t)$, and $c(\boldsymbol{x}, t)>c^{*}$ if $x>x_{t p}(t)$, but $c(\boldsymbol{x}, t) \leq c^{*}$ somewhere on the plane of $x=x_{t p}(t)$, respectively. Second, the leading $\left\{y=y_{l p}(t), z=z_{l p}(t)\right\}$ and trailing $\left\{y=y_{t p}(t), z=z_{t p}(t)\right\}$ points, characterized by the maximal and minimal, respectively, values of $c\left[x_{l p}(t), y, z, t\right]$ and $c\left[x_{t p}(t), y, z, t\right]$, respectively, were selected among all points on the leading and trailing planes, respectively.

Typical instantaneous flame shapes are shown in Figs. $2 \mathrm{a}$ and $2 \mathrm{~b}$ in $x y$ and $x z$-planes, respectively, where red filled circle indicates the leading point A. Instantaneous profiles of certain aforementioned quantities along lines $\left\{y=y_{l p}(t), z=z_{l p}(t)\right\},\left\{x=x_{l p}(t), z=z_{l p}(t)\right\}$, and $\left\{x=x_{l p}(t), y=y_{l p}(t)\right\}$, which are parallel to the $x, y$, and $z$-axes, respectively, are plotted in Figs. 3 and 4.

Figure 3a indicates that $B_{\boldsymbol{\omega} \cdot \boldsymbol{\omega}}>0$ in the leading point, see vertical dashed line, i.e., baroclinic torque locally increases the incoming enstrophy in this particular point. However, dashed blue line in Fig. 3a shows that $B_{\omega \cdot \omega}<0$ within the flamelet upstream of the leading point. Therefore, at low $c\left(x, y=y_{l p}, z=z_{l p}\right)$, an angle $\varphi$ between the vectors $B_{\boldsymbol{\omega}}$ and $\boldsymbol{\omega}$ is obtuse and the local enstrophy is reduced by baroclinic torque. When $c\left(x, y=y_{l p}, z=z_{l p}\right)$ increases with $x,|\varphi|$ is reduced and becomes equal to $\pi / 2$ at certain $c\left(x, y=y_{l p}, z=z_{l p}\right)$ upstream of the leading point $\boldsymbol{x}_{l p}(t)$ on the reaction surface. At larger $c\left(x, y=y_{l p}, z=z_{l p}\right)$, baroclinic torque increases $\omega^{2}$.

Red solid line in Fig. $3 \mathrm{~b}$ shows that $B_{\omega, y}(z)$ changes its sign in the vicinity of the leading point from positive at $z<z_{A}$ to negative at $z>z_{A}$, in line with the sketch in Fig. 1a. However, contrary 

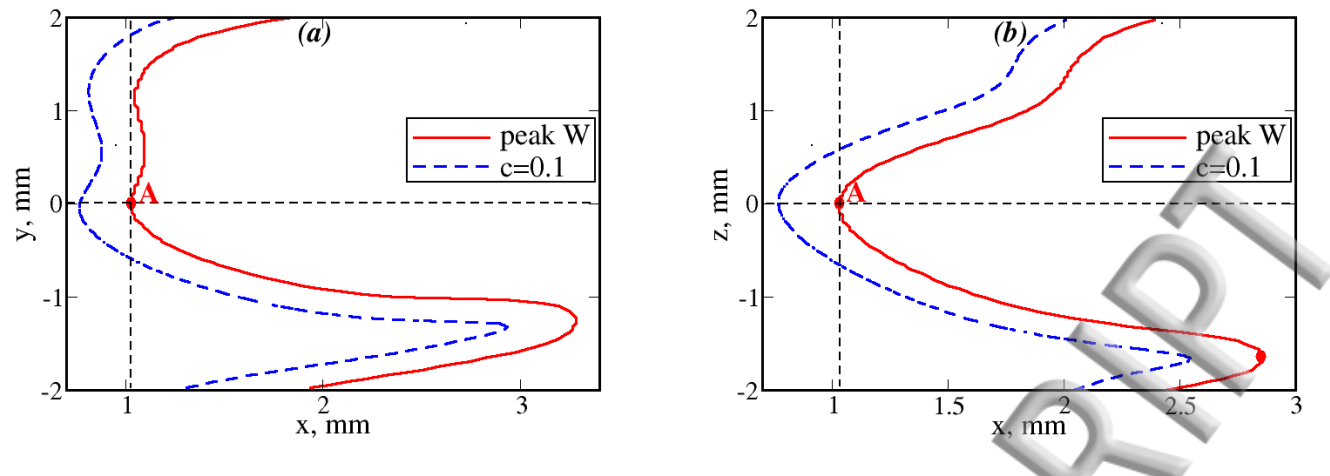

FIG. 2. Iso-lines of $c(x, y, z, t)=0.1$ (blue dashed lines) and $c(x, y, z, t)=0.85$ (red solid lines) on (a) $x y$ and (b) $x z$-planes at $t=10.5 \mathrm{~ms}$. Transverse distances $y$ and $z$ are counted from the leading point A. Case H.

to the sketch, $B_{\boldsymbol{\omega}, z}(y)$ is positive in the vicinity of the leading point, see blue dashed line, probably, because the reaction surface shown in Fig. 2a is weakly curved in the vicinity of point $A$ in the $x y$-plane. Nevertheless, $\partial B_{\omega, z} / \partial y>0$, in line with the sketch.

Red solid line in Fig. 4a indicates that $\omega_{y}(z)$ decreases with increasing $z$ in the vicinity of the point A due to the decrease in $B_{\boldsymbol{\omega}, y}(z)$ shown in red solid line in Fig. 3b. However, $\omega_{y}(z)$ does not change its sign in the point A, because the incoming $\omega_{y}>0$, see black dashed line in Fig. 4a. Therefore, counter-rotating vortex pair is not generated by $B_{\omega, y}$ in the $x z$-plane in this case. Nevertheless, such a vortex pair is observed in the $x y$-plane, as $\omega_{z}<0$ at $y<y_{A}$, but $\omega_{z}>0$ at $y>y_{A}$, see red solid line in Fig. 4b. Since the magnitude of the incoming $\omega_{z}$ is small, see black dashed line in Fig. $4 \mathrm{~b}$, the observed increase in $\omega_{z}$ with $y$ appears to be controlled by the aforementioned increase in $B_{\boldsymbol{\omega}, z}$ with $y$, which is consistent with the physical scenario sketched in Fig. 1.

Thus, the single-point, single-instant DNS data plotted in Figs. 3 and 4 are only partially consistent with that physical scenario. More specifically, the signs of $\partial B_{\omega, z} / \partial y, \partial B_{\omega, y} / \partial z, \partial \omega_{z} / \partial y$, and $\partial \omega_{y} / \partial z$ are consistent with the sketch, but the sign of (i) $B_{\boldsymbol{\omega}, z}(y)$ or (ii) $\omega_{y}(z)$ is not changed in the vicinity of the leading point. The matter is that, respectively, (i) the local $\nabla_{y} p$ multiplied with $\nabla_{x} \rho$, which magnitude $\left|\nabla_{x} \rho\right| \gg\left|\nabla_{y} \rho\right|$, can also contribute to $B_{\boldsymbol{\omega}, z}$ even if $\left|\nabla_{y} p\right| \ll\left|\nabla_{x} p\right|$ and 

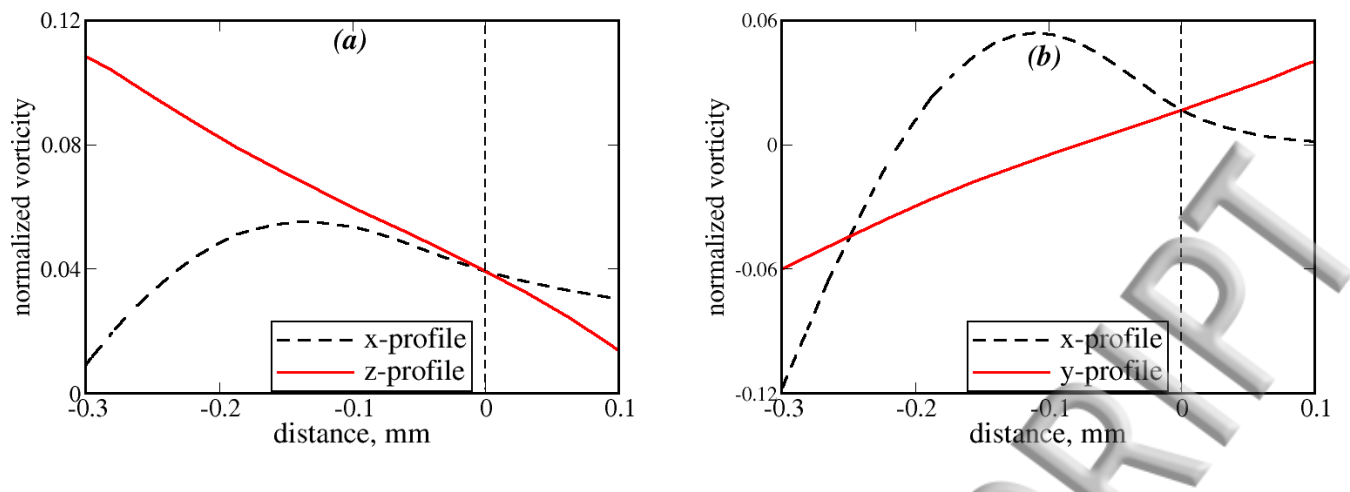

FIG. 4. Instantaneous profiles of (a) $\tau_{c} \omega_{y}$ and (b) $\tau_{c} \omega_{z}$ in the vicinity of the leading point A shown in Fig. 2. Local $x, y$, and $z$-distances are counted from the $x, y$, and $z$-coordinates, respectively, of the leading point.

(ii) $\omega_{y}(z)$ depends not only on $B_{\boldsymbol{\omega}, y}(z)$, but also on the incoming vorticity. Nevertheless, even in this partially consistent case, Fig. $4 \mathrm{~b}$ indicates generation of the counter-rotating vortex pair in the $x y$-plane, with this vortex pair pushing the leading point inside the mean flame brush, thus, reducing the reaction-surface area.

In order to statistically assess consistency of the physical scenario sketched in Fig. 1 with the DNS data computed in the vicinity of the leading and trailing points, the maximal and minimal values of $\omega_{y}\left(x_{A}, y_{A}, z\right)$ or $\omega_{z}\left(x_{A}, y, z_{A}\right)$ and $B_{\omega, y}\left(x_{A}, y_{A}, z\right)$ or $B_{\boldsymbol{\omega}, z}\left(x_{A}, y, z_{A}\right)$ were found within an interval of $\left(x_{A}, y_{A},\left|z-z_{A}\right|<\delta_{L}\right)$ or $\left(x_{A},\left|y-y_{A}\right|<\delta_{L}, z_{A}\right)$, respectively, at each time instant (221 and 200 instants in cases $\mathrm{H}$ and $\mathrm{L}$, respectively). Here, $A$ designates a leading or trailing point. Subsequent analysis of the obtained time series yielded the probabilities of various events that were directly associated with the physical scenario sketched in Fig. 1.

For the goals of the present work, the following four probabilities are of the most interest: (i) the probability $P_{\boldsymbol{\omega}}$ that a product of either $\max \left\{\omega_{y}\left(x_{A}, y_{A}, z\right)\right\}$ and $\min \left\{\omega_{y}\left(x_{A}, y_{A}, z\right)\right\}$ or $\max \left\{\omega_{z}\left(x_{A}, y, z_{A}\right)\right\}$ and $\min \left\{\omega_{z}\left(x_{A}, y, z_{A}\right)\right\}$ is negative, i.e., the probability that a transverse component of the vorticity vector changes its sign in the vicinity of the point $\mathrm{A}$;

(ii) the probability $P_{B}$ that a product of either $\max \left\{B_{\boldsymbol{\omega}, y}\left(x_{A}, y_{A}, z\right)\right\}$ and $\min \left\{B_{\boldsymbol{\omega}, y}\left(x_{A}, y_{A}, z\right)\right\}$ or $\max \left\{B_{\boldsymbol{\omega}, z}\left(x_{A}, y, z_{A}\right)\right\}$ and $\min \left\{B_{\boldsymbol{\omega}, z}\left(x_{A}, y, z_{A}\right)\right\}$ is negative, i.e., the probability that a transverse component of the baroclinic torque vector-term $\boldsymbol{B}_{\boldsymbol{\omega}}$ in Eq. (1) changes its sign in the vicinity of the point $\mathrm{A}$;

(iii) the probability $P_{y o r z}$ that (iii.a) either the $z$-coordinate $z_{\max }$ of $\max \left\{\omega_{y}\left(x_{A}, y_{A}, z\right)\right\}$ is smaller (larger) than the $z$-coordinate $z_{\min }$ of $\min \left\{\omega_{y}\left(x_{A}, y_{A}, z\right)\right\}$ in the vicinity of the leading (trailing) point or (iii.b) the $y$-coordinate $y_{\max }$ of $\max \left\{\omega_{z}\left(x_{A}, y, z_{A}\right)\right\}$ is larger (smaller) than the $y$-coordinate $y_{\min }$ of $\min \left\{\omega_{z}\left(x_{A}, y, z_{A}\right)\right\}$ in the vicinity of the leading (trailing) point, as such events are necessary in order for the counter-rotating vortex pairs sketched in Fig. 1 to reduce the reaction-surface area; and

(iv) the probability $P_{y a n d z}$ that both events (iii.a) and (iii.b) occur simultaneously.

Probabilities calculated for an iso-surface of $c(\boldsymbol{x}, t)=c^{*}=0.85$, associated with the peak local reaction rate, are reported in Table I. Note that the trends shown in Table I and discussed in the following are less pronounced if $c^{*}$ is decreased and the trends vanish if $c^{*}=0.1$.

Table I shows that, first, the probability $P_{\boldsymbol{\omega}}$ is equal or very close to unity, i.e., at least one of the transverse components of the vorticity vector changes its sign in the vicinity of the leading (or trailing) points. Second, the probability $P_{y o r z}$ is equal to unity for both leading and trailing points in cases $\mathrm{H}$ and $\mathrm{L}$, i.e. the local direction of an increase in either $\omega_{z}\left(x_{A}, y, z_{A}\right)$ or $\omega_{y}\left(x_{A}, y_{A}, z\right)$ is consistent with appearance of a vortex pair that pushes the leading (or trailing) point inside the mean flame brush. Even the probability $P_{y a n d z}$ is close to or equal to unity in the leading or trailing points in case $\mathrm{H}$. These features imply that the local vorticity field in the vicinity of the leading or trailing points works to smooth out the local reaction surface, thus, reducing its area. 
TABLE I. Probabilities of various events.

\begin{tabular}{ccccc}
\hline Probability & \multicolumn{2}{c}{ leading point } & \multicolumn{2}{c}{ trailing point } \\
& case $\mathrm{H}$ case $\mathrm{L}$ & case $\mathrm{H}$ & case $\mathrm{L}$ \\
\hline (i) $P_{\boldsymbol{\omega}}$ & 1.0 & 0.985 & 1.0 & 0.950 \\
(ii) $P_{\boldsymbol{B}}$ & 0.629 & 0.705 & 1.0 & 0.980 \\
(iii) $P_{\text {yorz }}$ & 1.0 & 1.0 & 1.0 & 1.0 \\
(iv) $P_{\text {yandz }}$ & 0.937 & 0.690 & 1.0 & 0.980 \\
\hline \hline
\end{tabular}

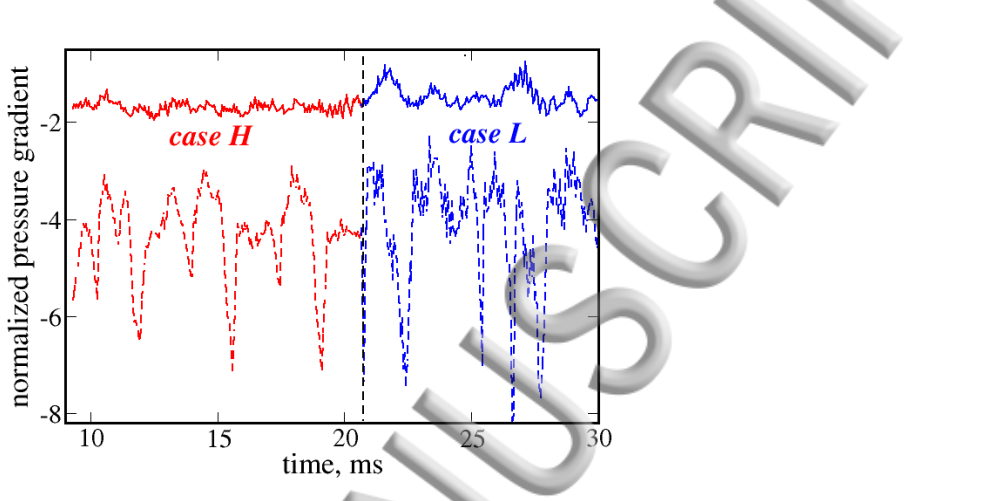

FIG. 5. Axial pressure gradient in the leading (solid lines) and trailing (dashed lines) points of the reaction surface of $c(\boldsymbol{x}, t)=0.85$ in cases $\mathrm{H}$ (red curves) and L (blue curves). The gradient is normalized using its value $\rho_{u}(\sigma-1) S_{L}^{2} / \delta_{L}$ associated with the unperturbed laminar premixed flame.

It is worth noting, however, that the probability $P_{B}$ is substantially less than unity at the leading points, thus, indicating that components $B_{z}\left(x_{A}, y, z_{A}\right)$ and $B_{y}\left(x_{A}, y_{A}, z\right)$ can retain the same sign for the entire intervals of $\left|y-y_{A}\right|<\delta_{L}$ and $\left|z-z_{A}\right|<\delta_{L}$, respectively. Typically, $\max \left\{B_{\boldsymbol{\omega}, y}\left(x_{A}, y_{A}, z\right)\right\}<$ 0 or $\min \left\{B_{\omega, y}\left(x_{A}, y_{A}, z\right)\right\}>0$ when a product of $\nabla_{x} \rho$ and $\nabla_{z} p$ overwhelms a product of $\nabla_{z} \rho$ and $\nabla_{x} p$ by virtue of $\left|\nabla_{x} \rho\right| \gg\left|\nabla_{z} \rho\right|$. Similarly, $\max \left\{B_{\boldsymbol{\omega}, z}\left(x_{A}, y, z_{A}\right)\right\}<0$ or $\min \left\{B_{\boldsymbol{\omega}, z}\left(x_{A}, y, z_{A}\right)\right\}>0$ when a product of $\nabla_{x} \rho$ and $\nabla_{y} p$ overwhelms a product of $\nabla_{y} \rho$ and $\nabla_{x} p$ by virtue of $\left|\nabla_{x} \rho\right| \gg\left|\nabla_{y} \rho\right|$. At the trailing points, the probability $P_{B}$ is equal or close to unity in case $\mathrm{H}$ or $\mathrm{L}$, respectively, because (i) the local pressure gradient in the vicinity of the trailing points is more affected by the combustioninduced thermal expansion when compared to the leading points, e.g. see Fig. 5, and (ii) baroclinic torque has directly and indirectly (due to viscous diffusion of vorticity generated within neighboring flamelet preheat zones) affected the local fluid particles during a longer time interval required for the particles to reach the trailing point.

The difference between the values of $P_{\boldsymbol{\omega}}$ and $P_{B}$, reported in Table I, is associated with the influence of baroclinic torque on the vorticity field upstream of the leading points on the reaction surface of $c(\boldsymbol{x}, t)=0.85$. Indeed, while the probabilities $P_{B}$ are almost the same $(0.58,0.58,0.56$, and 0.58 ) for $c^{*}=0.1,0.25,0.50$, and 0.75 , respectively, in case $\mathrm{H}$, the probabilities $P_{\boldsymbol{\omega}}$ are significantly different, i.e., $0.69,0.96,1.0$, and 1.0 , respectively, thus, indicating that the evolution of the local vorticity field from the flamelet cold edges to the reaction zones facilitates appearance of the aforementioned counter-rotating vortex pair upstream of the leading point on the reaction surface.

On the contrary, in case L, the probabilities $P_{\boldsymbol{\omega}}$ are almost the same for $c^{*}=0.1,0.25,0.50$, and 0.75 , respectively, i.e., the probability does not change within flamelets. The point is that the magnitude of baroclinic torque is much less in this case, cf. figures 9 and 10 in an earlier paper, ${ }^{42}$ due to a significantly lower density ratio. Accordingly, the influence of the baroclinic torque on the vorticity field is much weaker pronounced in case $\mathrm{L}$ when compared to case $\mathrm{H}$, as discussed in detail elsewhere. ${ }^{42}$

Finally, to estimate the order of magnitude of the axial velocity $u_{r o t}$ induced by the aforemen- 


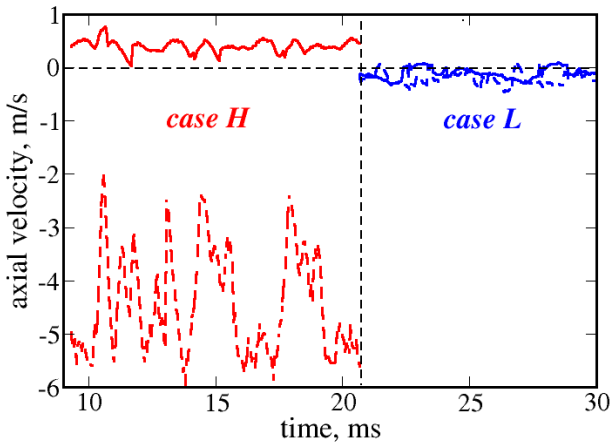

FIG. 6. Axial velocity estimated using Eq. (5) in the leading (solid lines) and trailing (dashed lines) points on the reaction surface of $c(\boldsymbol{x}, t)=0.85$ in cases $\mathrm{H}$ (red curves) and $\mathrm{L}$ (blue curves).

tioned counter-rotating vortex pair, the following quantity

$$
\begin{aligned}
u_{\text {rot }}= & -\max \left\{\omega_{y}\left(x_{A}, y_{A}, z\right)\right\}\left(z_{\max }-z_{A}\right)+\min \left\{\omega_{y}\left(x_{A}, y_{A}, z\right)\right\}\left(z_{A}-z_{\min }\right) \\
& +\max \left\{\omega_{z}\left(x_{A}, y_{1} z_{A}\right)\right\}\left(y_{\max }-y_{A}\right)-\min \left\{\omega_{z}\left(x_{A}, y, z_{A}\right)\right\}\left(y_{A}-y_{\min }\right)
\end{aligned}
$$

was evaluated in the leading and trailing points. Four terms in this equation are associated with the axial velocity differences (i) $u\left(x_{A}, y_{A}, z_{A}\right)-u\left(x_{A}, y_{A}, z_{\max }\right)$ due to vorticity $\omega_{y}\left(x_{A}, y_{A}, z_{\max }\right)$, which involves $\partial u / \partial z$, (ii) $u\left(x_{A}, y_{A}, z_{A}\right)-u\left(x_{A}, y_{A}, z_{\min }\right)$ due to vorticity $\omega_{y}\left(x_{A}, y_{A}, z_{\min }\right)$, (iii) $u\left(x_{A}, y_{A}, z_{A}\right)-$ $u\left(x_{A}, y_{\max }, z_{A}\right)$ due to vorticity $\omega_{z}\left(x_{A}, y_{\max }, z_{A}\right)$, which involves $-\partial u / \partial y$, and (iv) $u\left(x_{A}, y_{A}, z_{A}\right)-$ $u\left(x_{A}, y_{\min }, z_{A}\right)$ due to vorticity $\omega_{z}\left(x_{A}, y_{\min }, z_{A}\right)$. In sketches shown in Fig. 1a (1b), each of these four terms should be positive (negative, respectively).

The results obtained for the reaction surface of $c(x, t)=0.85$ and plotted in Fig. 6 show that this velocity works to move the leading and trailing points inward the mean flame brush in case H, i.e., $u_{r o t}>0$ in the leading points and $u_{r o t}<0$ in the trailing points, with the magnitude of $u_{r o t}$ being comparable with $S_{L}, u^{\prime}$, and $\overline{S_{T}}$ in the leading points and being much larger than $S_{L}, u^{\prime}$, and $\overline{S_{T}}$ in the trailing points. In case $\mathrm{L}$ associated with a low density ratio and a weak influence of baroclinic torque on the vorticity field, the magnitude of $u_{r o t}$ is much less, there is a significant probability of $u_{r o t}<0$ in the leading point, but $u_{r o t}$ is predominantly positive in the trailing point, thus, implying some weak influence of baroclinic torque.

Thus, the above analysis of statistical characteristics of the flow field within flamelets in the vicinity of the leading and trailing points on the reaction surface indicates that, in case $\mathrm{H}$ characterized by a large density ratio, baroclinic torque works to push these points inward the mean flame brush, thus, reducing the reaction-surface area, with the effect being significantly more pronounced in the vicinity of the trailing points. In case $\mathrm{L}$ characterized by a low density ratio, baroclinic torque plays a less important role. In the next subsection, statistical results extracted from the entire flame brush are considered.

\section{III.B. Conditioned statistics of stretch rate}

Since the rate of an increase (or decrease) in the local area $A^{*}$ of an iso-scalar surface of $c(\boldsymbol{x}, t)=$ $c^{*}$ is well known ${ }^{38,86-88}$ to be controlled by the local stretch rate $\dot{s}=\nabla \cdot \boldsymbol{u}-\boldsymbol{n n}: \boldsymbol{u}+S_{d} \nabla \boldsymbol{n}$, i.e., $d \ln A^{*} / d t=\dot{s}$, the focus of the following discussion will be placed on the joint statistics of $\dot{s}$ and $\boldsymbol{\omega}, \omega^{2}, B_{\boldsymbol{\omega}}$, or $B_{\boldsymbol{\omega} \cdot \boldsymbol{\omega}}$. Here, $\boldsymbol{n}=-\nabla c /|\nabla c|$ is the unit vector normal to the iso-scalar surface, $S_{d}=[\nabla \cdot(\rho D \nabla c)+W] /(\rho|\nabla c|)$ is the local displacement speed, $D$ is the molecular diffusivity of $c$, and $W$ is the mass rate of product creation.

Figure 7 shows that the probability of negative (black solid lines) stretch rates is higher than the probability of $\dot{s}>0$ (red dashed lines) in regions characterized by a large magnitude of the baroclinic torque term $B_{\boldsymbol{\omega} \cdot \boldsymbol{\omega}}(\boldsymbol{x}, t)$ in the $\omega^{2}$-transport Eq. (3). On the contrary, relation between the 

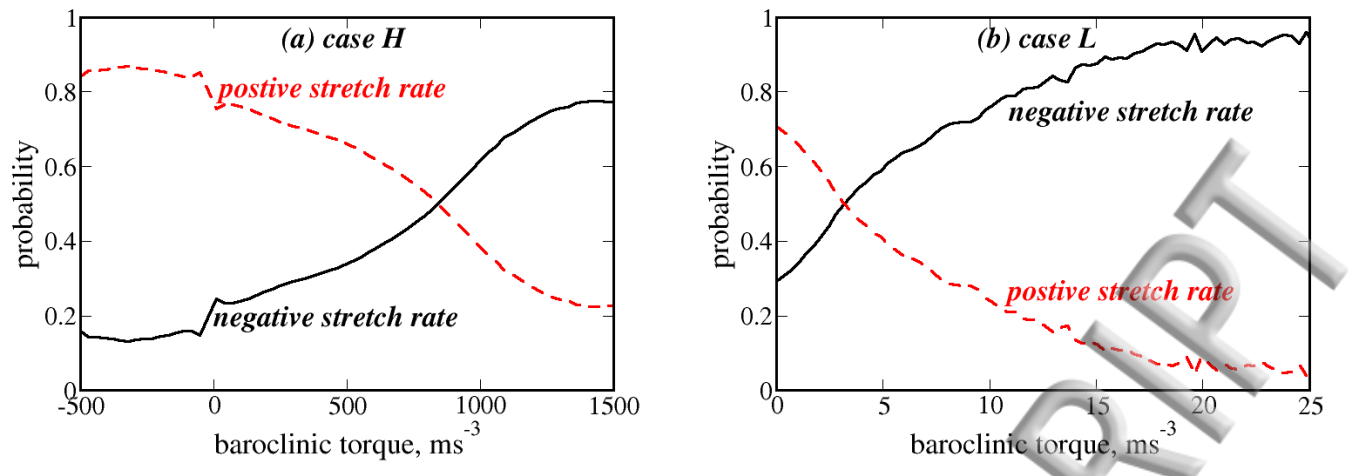

FIG. 7. Probabilities of positive (red dashed lines) and negative (black solid lines) stretch rates conditioned to the local value of baroclinic torque term $B_{\boldsymbol{\omega} \cdot \boldsymbol{\omega}}(\boldsymbol{x}, t)$ in the transport equation for enstrophy. (a) case $\mathrm{H},(\mathrm{b})$ case L.

two computed probabilities is well known to be opposite if they are extracted from the entire flame brush and the present DNS data do show that opposite relation, e.g., see Fig. 10 discussed later. Since $d \ln A^{*} / d t=\dot{s}$, Fig. 7 indicates that the area of a surface of $c(\boldsymbol{x}, t)=c^{*}$ is statistically reduced in regions associated with the strongest generation of enstrophy due to baroclinic torque.

Such a trend is not observed within flamelet preheat zone $(c<0.65)$, where the local stretch rates are predominantly positive, e.g., see Fig. 8a, but is well pronounced in the vicinity of the reaction zone $(c>0.65)$, where $\dot{s}(\boldsymbol{x}, t)$ is predominantly negative provided that $B_{\boldsymbol{\omega} \cdot \boldsymbol{\omega}}(\boldsymbol{x}, t)$ is sufficiently large, e.g., see Fig. 8b. Even at the reaction zone, the trend is not pronounced at the leading edge of the mean flame brush, e.g., see Fig. 9a, but is well pronounced in the middle of the flame brush, e.g., see Fig. $9 \mathrm{~b}$, or at larger $\bar{c}(x)$.

On the contrary, the same DNS data conditioned solely (independently of $B_{\boldsymbol{\omega} \cdot \boldsymbol{\omega}}$ ) to various local $c(\boldsymbol{x}, t)$, see Fig. 10, show well-known predominance of positive stretch rates in the middle of the flame brush, i.e., at $\bar{c}=0.5$. Moreover, in case H, Fig. 10a indicates an increase in the probability of finding $\dot{s}(\boldsymbol{x}, t)<0$ with increasing the local $c(\boldsymbol{x}, t)$, which the data are conditioned to, but such a trend is not observed in case L, see Fig. 10b. This difference between results computed in the two cases is consistent with significant (negligible) generation of vorticity by large (small, respectively, cf. abscissa coordinates in Figs. 7a and 7b, respectively) baroclinic torque term in case $\mathrm{H}$ (L, 

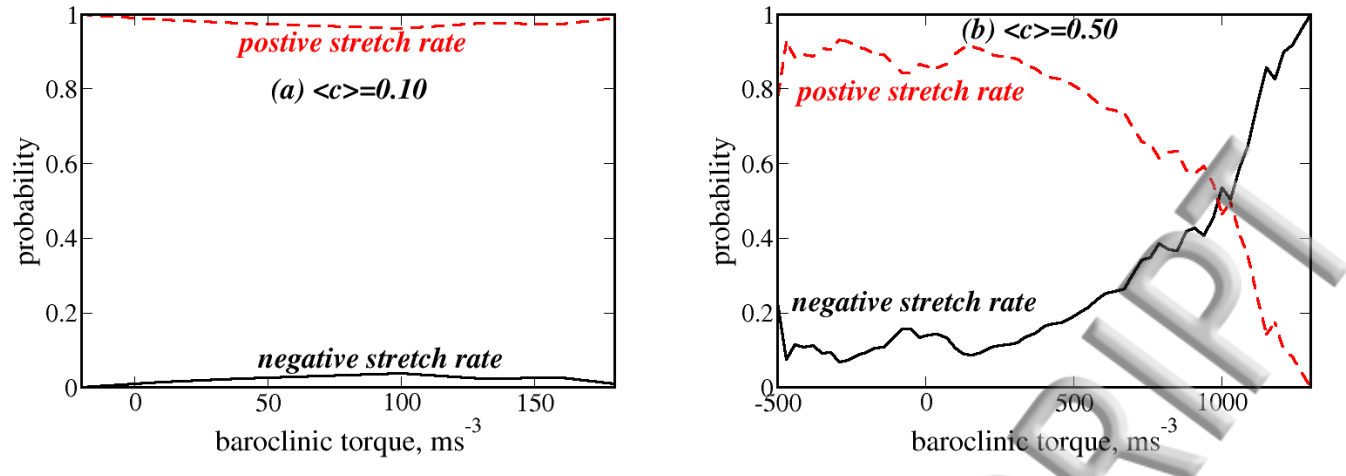

FIG. 9. Probabilities of positive (red dashed lines) and negative (black solid lines) stretch rates doubly conditioned to (i) the local value of baroclinic torque term $B_{\boldsymbol{\omega} \cdot \boldsymbol{\omega}}(\boldsymbol{x}, t)$ in the transport equation for enstrophy and (ii) the local value of the combustion progress variable $0.65<c(\boldsymbol{x}, t)<0.75$. The probabilities are evaluated in two different regions of the mean flame brush. (a) $0.05<\bar{c}(x)<0.15$, (b) $0.45<\bar{c}(x)<0.55$. Case H. Results obtained in case $\mathrm{L}$ are qualitatively similar.

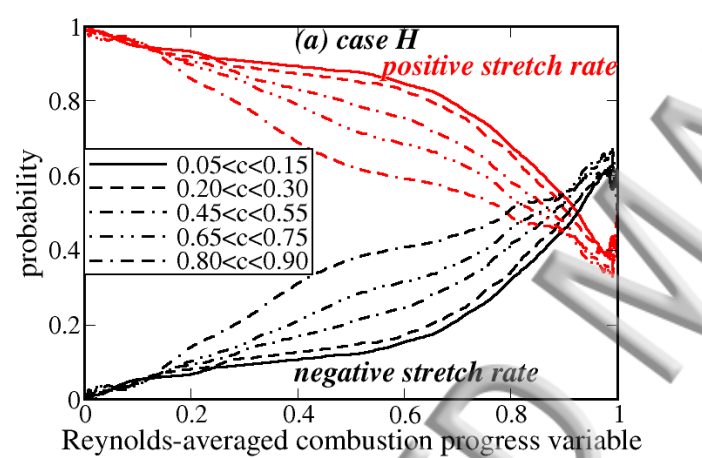

Reynolds-averaged combustion progress variable

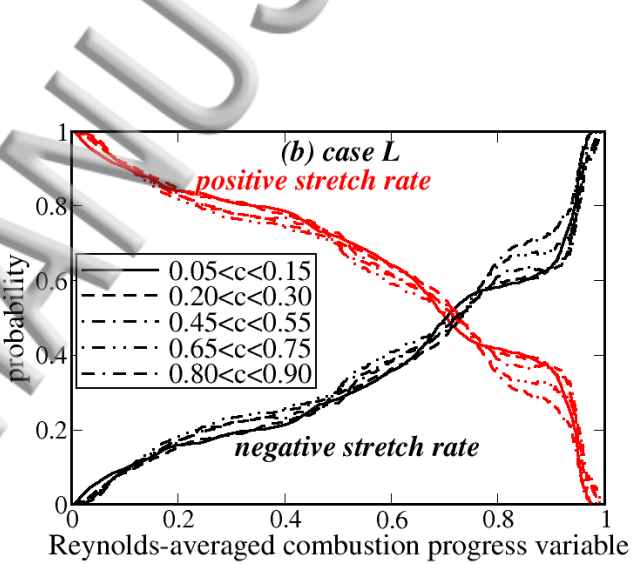

Reynolds-averaged combustion progress variable

FIG. 10. Probabilities of positive (red lines) and negative (black lines) stretch rates conditioned to five different intervals of the local value of the combustion progress variable $c(\boldsymbol{x}, t)$, specified in legends. (a) case $\mathrm{H}$, (b) case L.

respectively), as the vorticity generated by $B_{\boldsymbol{\omega} \cdot \boldsymbol{\omega}}(\boldsymbol{x}, t)$ works to generate negative stretch rates.

At first glance, the results plotted in Fig. 9a may appear to be inconsistent with the contents of Section III.A, where reduction of the reaction-surface area due to the flame-generated vorticity was discussed by analyzing the DNS data obtained at the leading edge, i.e., at a low $\bar{c}(x)$. However, it is worth remembering that the local stretch rate is affected not only by the local vorticity and baroclinic torque, but also by the potential component of the local velocity field. Accordingly, even if the flame-generated vorticity works to reduce the local reaction-surface area, such a reduction effect can be overwhelmed due to stretching of the surface by the potential component of the velocity field. Only if the former (reduction) effect is sufficiently strong, it can locally overwhelm the latter effect, but a sufficiently large $\bar{c}$ is required in order for this to occur.

Thus, the discussed reduction effect of baroclinic torque on the rate $d \ln A^{*} / d t$ of an increase in the area of an iso-scalar surface of $c(x, t)=c^{*}$ is enhanced both by $c^{*}$ and by $\bar{c}(x)$. The former trend can be attributed to shortage of time during that the baroclinic torque has affected fluid particles that reach an iso-scalar surface characterized by a low $c^{*}$. The letter trend can be attributed to an increase in the magnitude of $B_{\boldsymbol{\omega} \cdot \boldsymbol{\omega}}$ with increasing $\bar{c}(x)$, e.g., cf. abscissa coordinates in Figs. 9a and 9b.

Figure 11a shows that, in case $\mathrm{H}$, the probability of negative (black solid line) stretch rates is 

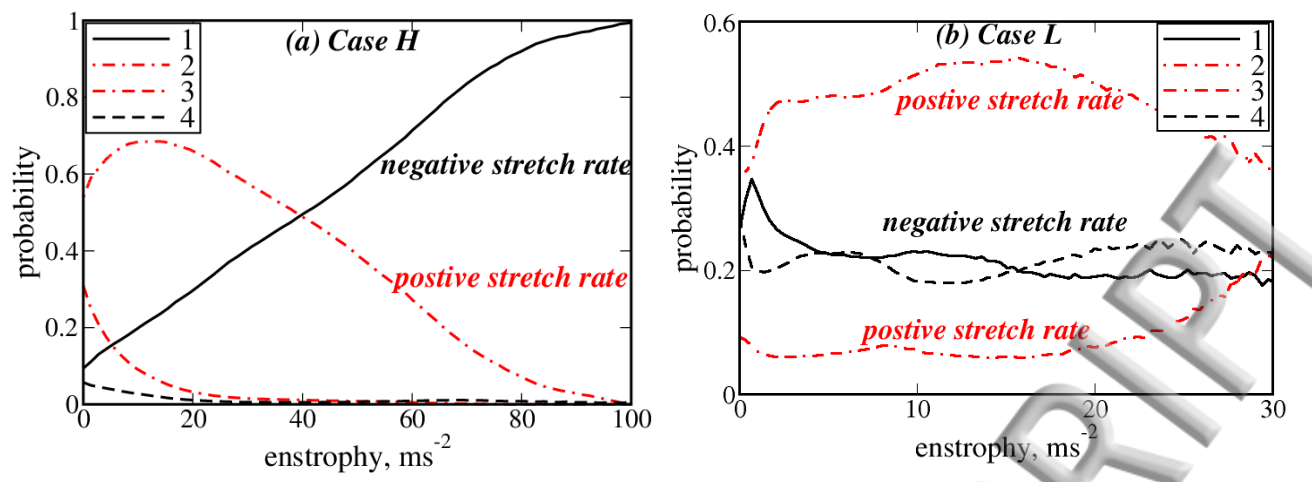

FIG. 11. Probabilities of positive (red dotted-dashed lines 2 and 3) and negative (black solid and dashed lines 1 and 4) stretch rates conditioned to the local value of enstrophy $\omega^{2}(\boldsymbol{x}, t)$. Curves 1-2 and 3-4 are associated with the positive and negative, respectively, baroclinic torque term $B \boldsymbol{\omega} \cdot \omega(\boldsymbol{x}, t)$. (a) case H, (b) case L.

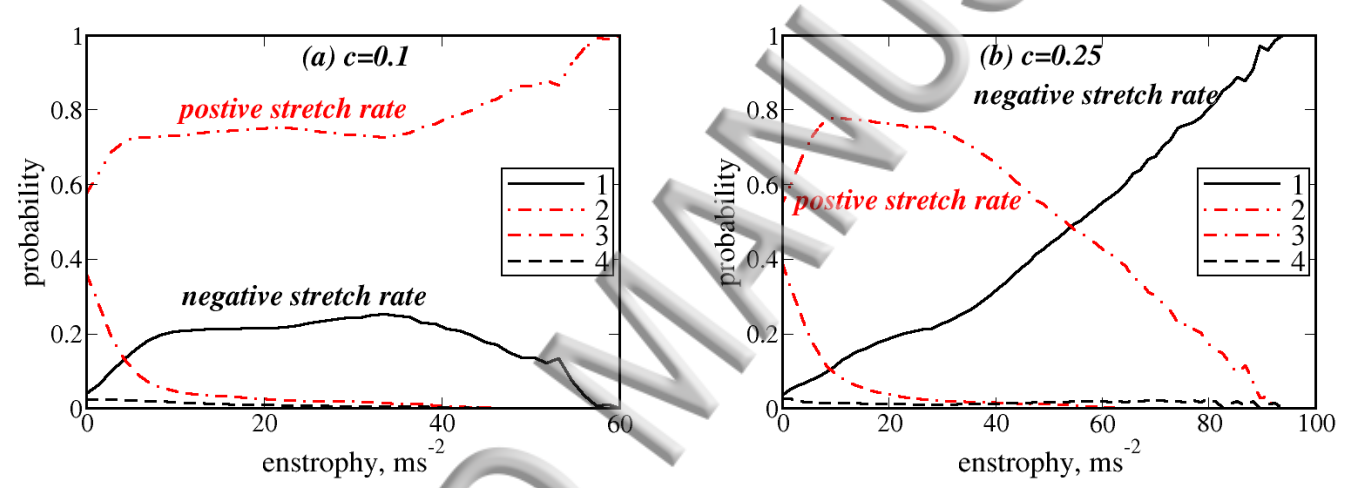

FIG. 12. Probabilities of positive (red dotted-dashed lines 2 and 3) and negative (black solid and dashed lines 1 and 4) stretch rates doubly conditioned to (i) the local value of enstrophy $\omega^{2}(\boldsymbol{x}, t)$ and (ii) the local value of the combustion progress yariable. (a) $0.05<c(\boldsymbol{x}, t)<0.15$, (b) $0.20<c(\boldsymbol{x}, t)<0.30$. Curves $1-2$ and 3-4 are associated with the positive and negative, respectively, baroclinic torque term $B_{\boldsymbol{\omega} \cdot \boldsymbol{\omega}}(\boldsymbol{x}, t)$. Case H.

higher than the probability of $\dot{s}(\boldsymbol{x}, t)>0$ (red dotted-dashed lines) in regions characterized by a large enstrophy $\omega^{2}(\boldsymbol{x}, t)$. A similar trend was already discussed for regions characterized by a large magnitude of the baroclinic torque term $B_{\boldsymbol{\omega} \cdot \boldsymbol{\omega}}(\boldsymbol{x}, t)$ in the $\omega^{2}$-transport Eq. (3), see Fig. 7a.

However, in case $\mathrm{L}$, the results plotted in Fig. 11b differ significantly from the results shown in Fig. $7 b$, i.e., the former figure shows that the stretch rates are predominately positive independently on $\omega^{2}$. Predominance of positive stretch rates is also observed for data conditioned solely (independently of $\left.\omega^{2}\right)$ to various local $c(\boldsymbol{x}, t)$ and extracted from various regions of the mean flame brush, with exception of its trailing zone ( $\bar{c}>0.7)$, where the flame surface area is consumed, see Fig. 10b. The difference between the results plotted in Figs. $7 \mathrm{~b}$ and $11 \mathrm{~b}$ is associated with the fact that the magnitude of $B_{\boldsymbol{\omega} \cdot \boldsymbol{\omega}}$ is much less in case $\mathrm{L}$ when compared to case $\mathrm{H}$, cf. abscissa coordinates in Figs. $7 \mathrm{a}$ and $7 \mathrm{~b}$. Accordingly, baroclinic torque barely affects the vorticity field in case $\mathrm{L}$ and the enstrophy decays within the mean flame brush. ${ }^{42}$ Thus, the flame-generated vorticity is weak and plays a minor role in case L. This explanation is consistent with Fig. 10b, which does not reveal any dependence of the probability of finding $\dot{s}(\boldsymbol{x}, t)<0$ on the local $c(\boldsymbol{x}, t)$ in case $\mathrm{L}$.

On the contrary, in case $\mathrm{H}$, the flame-generated vorticity can play a substantial role and, in particular, (i) is associated with a well pronounced increase in the probability of finding $\dot{s}(\boldsymbol{x}, t)<0$ with increasing the local $c(\boldsymbol{x}, t)$, see Fig. 10a, and (ii) yields negative stretch rates in regions character- 

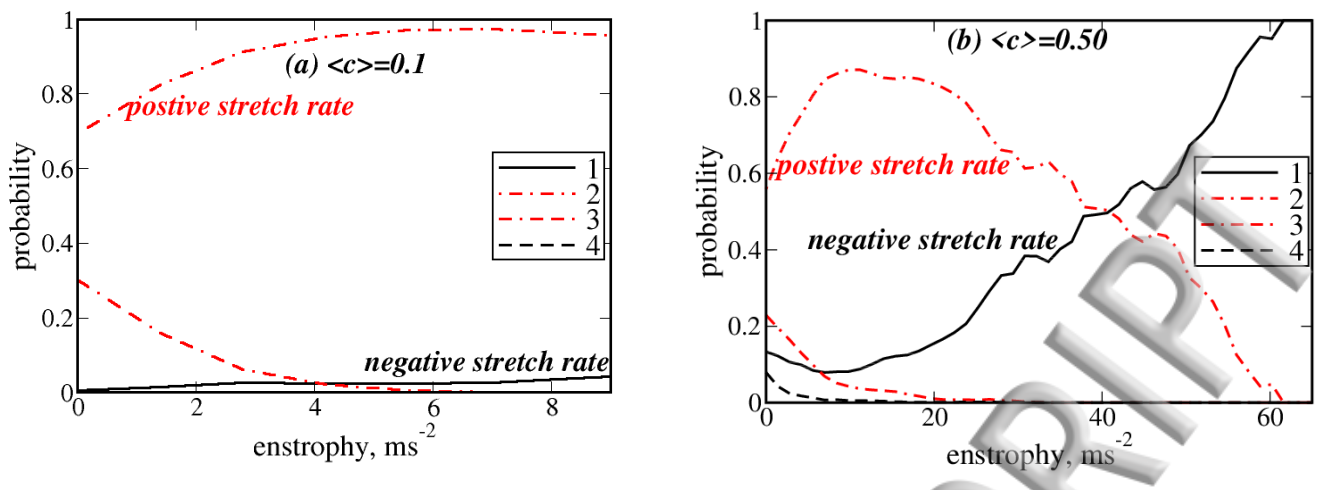

FIG. 13. Probabilities of positive (red dotted-dashed lines 2 and 3) and negative (black solid and dashed lines 1 and 4) stretch rates doubly conditioned to (i) the local value of enstrophy $\omega^{2}(\boldsymbol{x}, t)$ and (ii) the local value of the combustion progress variable $0.65<c(\boldsymbol{x}, t)<0.75$. The probabilities are evaluated in two different regions of the mean flame brush. (a) $0.05<\bar{c}(x)<0.15$, (b) $0.45<\bar{c}(x)<0.55$. Curves $1-2$ and 3-4 are associated with the positive and negative, respectively, baroclinic torque term $B \boldsymbol{\omega} \cdot \boldsymbol{\omega}(\boldsymbol{x}, t)$. Case $\mathrm{H}$.

ized by a large $\omega^{2}(\boldsymbol{x}, t)$, as indicated in Fig. 11a. Figures 12 and 13 show that the trend is more pronounced at larger $c^{*}$ and larger $\bar{c}(x)$, similarly to the already discussed correlation between the sign of $\dot{s}(\boldsymbol{x}, t)$ and the magnitude of $B_{\boldsymbol{\omega} \cdot \boldsymbol{\omega}}(\boldsymbol{x}, t)$. Note that, in the case of enstrophy, the stretch rate is predominantly negative at large $\omega^{2}$ not only in the reaction zone, but also in the preheat zone, see Fig. 12b.

To evaluate the contribution of regions characterized by a large magnitude of $\omega^{2}(\boldsymbol{x}, t)$ or $B_{\boldsymbol{\omega} \cdot \boldsymbol{\omega}}(\boldsymbol{x}, t)$ to the evolution of the local areas of various iso-scalar surfaces of $c(\boldsymbol{x}, t)=c^{*}$, the following two quantities were computed when processing the DNS data. First, the mean rate of an increase in the surface area, conditioned to $c_{1}<c(x, t)<c_{2}$ and conditionally averaged in volumes characterized by $q_{1}<q(\boldsymbol{x}, t)<q_{2}$, was calculated as follows

$$
\left\langle\frac{d \Sigma}{d t} \mid c_{1}<c<c_{2}, q_{1}<q<q_{2}\right\rangle=\frac{\int_{t_{2}}^{t_{3}} \iiint \dot{s}|\nabla c| \Pi\left(c_{1}<c<c_{2}\right) \Pi\left(q_{1}<q<q_{2}\right) d \boldsymbol{x} d t}{\int_{t_{2}}^{t_{3}} \iiint \Pi\left(c_{1}<c<c_{2}\right) \Pi\left(q_{1}<q<q_{2}\right) d \boldsymbol{x} d t} .
$$

Here, $q$ designates either enstrophy $\omega^{2}$ or baroclinic torque $B_{\boldsymbol{\omega} \cdot \boldsymbol{\omega}}$, the difference $\Pi\left(q_{1}<q<q_{2}\right) \equiv$ $H\left(q-q_{2}\right)-H\left(q-q_{1}\right)$ between Heaviside functions $H(q)$ is equal to unity if $q_{1}<q<q_{2}$ and vanishes otherwise, $\Sigma=|\nabla c|$ is the flame surface density, ${ }^{57} t_{2}$ and $t_{3}$ are boundaries of the time interval during that the mean inlet velocity is constant and the DNS data are analyzed, see Section II, and the integral in the numerator characterizes the rate of an increase in the surface area, because, for an infinitesimal volume $d V$, the local area $\delta A^{*}=\Sigma^{*} d V$ and $d\left(\delta A^{*}\right) / d t=\dot{s} \delta A^{*}=\dot{s}|\nabla c|_{c=c^{*}} d V$.

Figures $14 \mathrm{a}$ and $15 \mathrm{a}$ show that, both in the reaction zone (see red solid lines) and in the middle of flamelets (orange dotted-dashed lines) in case $\mathrm{H}$, the doubly conditioned rate given by Eq. (6) is negative in regions characterized by a large $B_{\boldsymbol{\omega} \cdot \boldsymbol{\omega}}(\boldsymbol{x}, t)$ or a large $\omega^{2}(\boldsymbol{x}, t)$, respectively. Thus, Figs. $14 \mathrm{a}$ and $15 \mathrm{a}$ further support the finding that the flame-generated vorticity can work to impede increasing the flame-surface area. The same trend is observed for the reaction zone and large $B_{\omega \cdot \omega}(\boldsymbol{x}, t)$ in case L, see red solid line in Fig. $14 \mathrm{~b}$, but the discussed rate is positive in regions characterized by a large enstrophy $\omega^{2}(x, t)$ in case L, see Fig. $15 \mathrm{~b}$. The latter trend is associated with a weak influence of baroclinic torque on the enstrophy in case $\mathrm{L}$, as discussed earlier.

Second, a relative mean bulk rate of an increase in the surface area in volumes characterized by $q_{1}<q(\boldsymbol{x}, t)<q_{2}$ when compared to the entire flame brush was evaluated as follows

$$
\left\langle\delta A_{F} \mid c_{1}<c<c_{2}, q_{1}<q<q_{2}\right\rangle=\frac{\int_{t_{2}}^{t_{3}} \iiint \dot{s}|\nabla c| \Pi\left(c_{1}<c<c_{2}\right) \Pi\left(q_{1}<q<q_{2}\right) d \boldsymbol{x} d t}{\int_{t_{2}}^{t_{3}} \iiint \dot{s}|\nabla c| \Pi\left(c_{1}<c<c_{2}\right) d \boldsymbol{x} d t} .
$$

Figures 16a and 17a show that, both in the reaction zone (see red solid lines) and in the middle of flamelets (orange dotted-dashed lines) in case $\mathrm{H}$, the relative mean bulk rate given by Eq. (7) is negative in regions characterized by a large $B_{\boldsymbol{\omega} \cdot \boldsymbol{\omega}}(\boldsymbol{x}, t)$ or a large $\omega^{2}(\boldsymbol{x}, t)$, respectively, with 

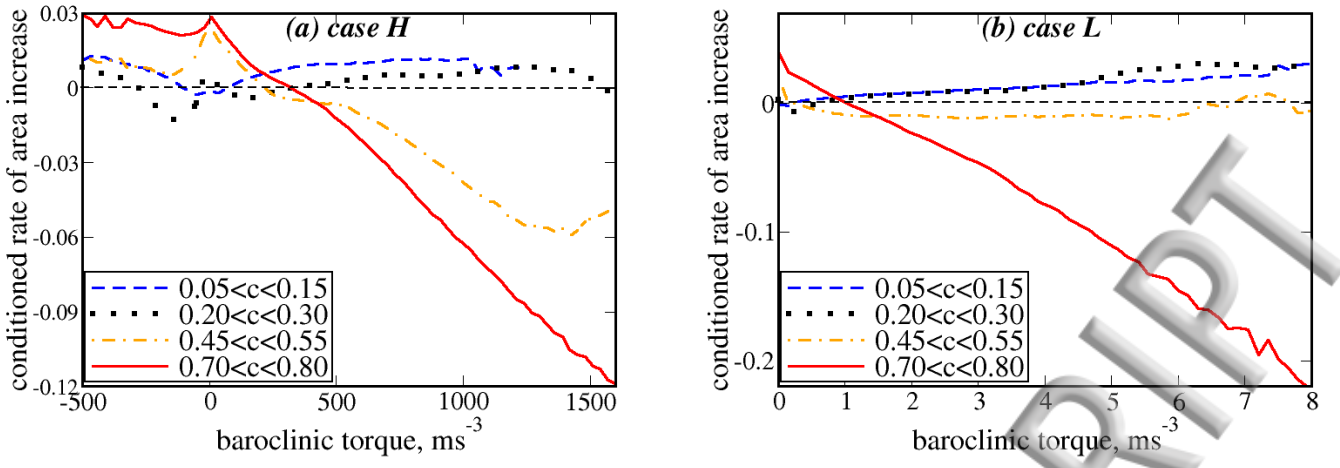

FIG. 14. Doubly conditioned rate of an increase in flame surface area, given by Eq. (6) and normalized using $\tau_{c} \delta_{L}$, vs. the baroclinic torque term $B_{\boldsymbol{\omega} \cdot \boldsymbol{\omega}}$ in the enstrophy transport equation. Intervals $c_{1}<c(\boldsymbol{x}, t)<c_{2}$ that the rate is conditioned to are specified in the legends. (a) case H, (b) case L.
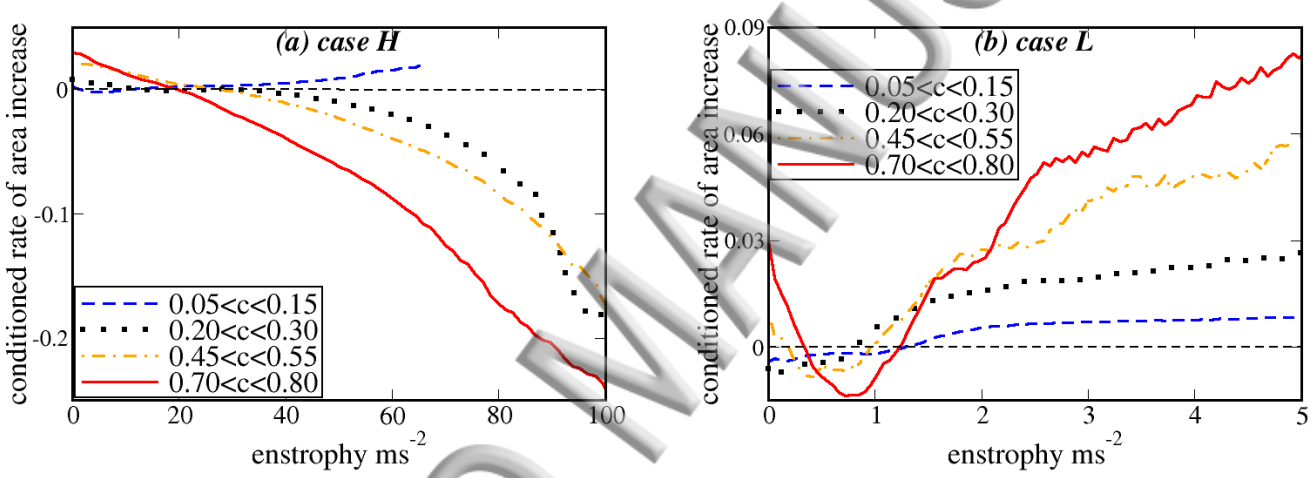

FIG. 15. Doubly conditioned rate of an increase in flame surface area, given by Eq. (6) and normalized using $\tau_{c} \delta_{L}$, vs. enstrophy $\omega^{2}$. Intervals $c_{1}<c(x, t)<c_{2}$ that the rate is conditioned to are specified in the legends. (a) case $\mathrm{H}$, (b) case L.

the magnitude of this negative rate being substantial. Thus, the reduction of the reaction-surface area (see red solid lines) by the flame-generated vorticity plays a substantial role in case H. On the contrary, in case L characterized by a low density ratio, this physical mechanism is of minor importance, see Figs. $16 \mathrm{~b}$ and $17 \mathrm{~b}$. Even if the relative mean bulk rate conditioned to the reaction zone is negative in regions characterized by a large $B_{\boldsymbol{\omega} \cdot \boldsymbol{\omega}}(\boldsymbol{x}, t)$ in case $\mathrm{L}$ (see red solid line in Fig. $16 \mathrm{~b}$ ), the magnitude of this rate is very low due to a weak influence of baroclinic torque on the velocity field in case $\mathrm{L}$, as discussed earlier.

It is of interest to note that the rates given by Eqs. (6) and (7) and conditioned to the cold regions of flamelet preheat zones are statistically negative if the local magnitude of $B_{\boldsymbol{\omega} \cdot \boldsymbol{\omega}}(\boldsymbol{x}, t)$ or $\omega^{2}(\boldsymbol{x}, t)$ is small, see dashed violet lines in Figs. 14-17. This effect is beyond the scope of the present study and will be examined in a future paper.

\section{. III.C. Vorticity transformation upstream of flamelets}

In the constant-density flow of the unburned reactants upstream of flamelets, the baroclinic torque terms $\boldsymbol{T}_{4}$ and $T_{4}$ vanish in Eqs. (1) and (3), respectively, as well as the dilatation terms $\boldsymbol{T}_{3}$ and $T_{3}$, respectively. Nevertheless, thermal expansion in a flame could indirectly affect vorticity in the incom- 

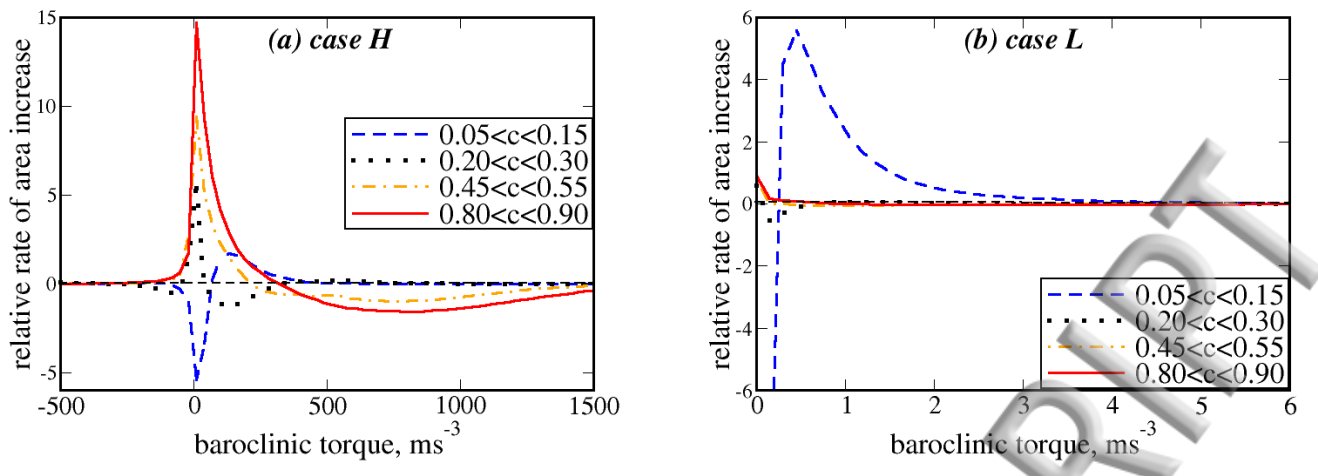

FIG. 16. Doubly conditioned relative mean bulk rate of an increase in flame surface area, given by Eq. (7), vs. the baroclinic torque term $B_{\boldsymbol{\omega} \cdot \boldsymbol{\omega}}$ in the enstrophy transport equation. Intervals $c_{1}<c(\boldsymbol{x}, t)<c_{2}$ that the rate is conditioned to are specified in the legends. (a) case H, (b) case L.
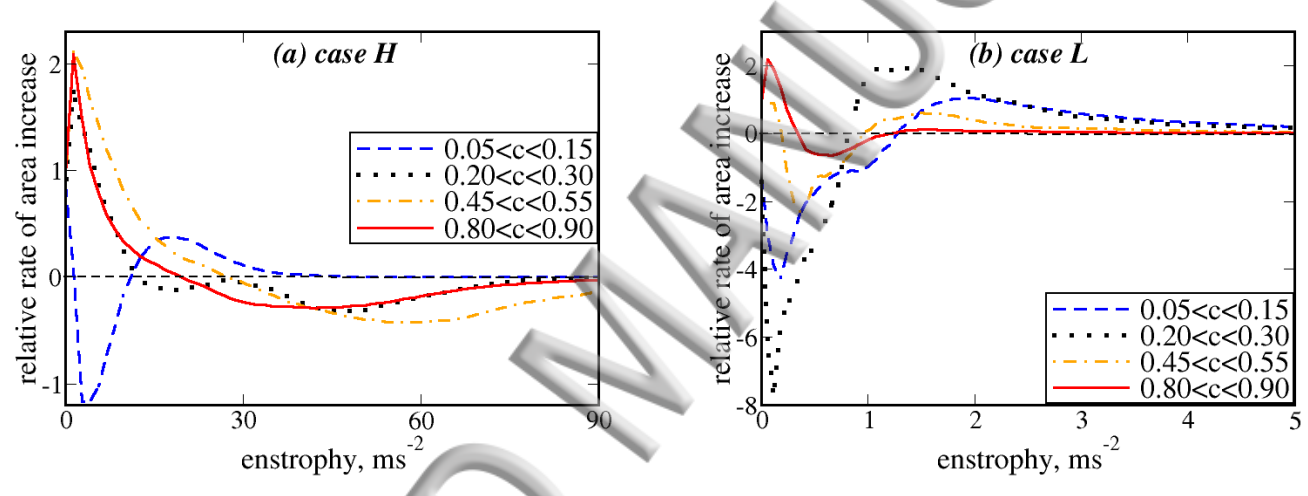

FIG. 17. Doubly conditioned relative mean bulk rate of an increase in flame surface area, given by Eq. (7), vs. enstrophy $\omega^{2}$. Intervals $c_{1}<c(\boldsymbol{x}, t)<c_{2}$ that the rate is conditioned to are specified in the legends. (a) case $\mathrm{H}$, (b) case L.

ing turbulent flow of the unburned reactants, because combustion-induced pressure perturbations cause potential velocity perturbations in the incoming flow, thus, changing the vortex-stretching terms $\boldsymbol{T}_{1}$ and $T_{1}$ and the dissipation terms $\boldsymbol{T}_{2}$ and $T_{2}$.

Some effects due to such pressure and potential velocity perturbations in the unburned reactants are well known. For instance, the Darrieus-Landau (DL) physical mechanism, i.e., acceleration of the constant-density flow of the unburned reactants by the combustion-induced pressure perturbations, causes the hydrodynamic instability of laminar premixed flames ${ }^{37}$ or growth of unburned mixture fingers in turbulent premixed flames, with the latter phenomenon being revealed ${ }^{65,71}$ by analyzing the present DNS data. However, the DL mechanism and the phenomena governed by it are fundamentally different from the physical mechanisms and phenomena studied in the present paper. Indeed, the latter are governed by baroclinic torque, which vanishes in the constant-density flow of the unburned reactants. We may also note that the flame-generated vorticity works to smooth out the surface of an unstable laminar premixed flame, as discussed in Section I. In a turbulent flame, the decrease in the flame-surface area by the flame-generated vorticity can counteract the DL mechanism. Such a counteraction can, in part, explain why the ratio of $\overline{S_{T}} / S_{L}$ is almost the same in cases $\mathrm{H}$ and L, see Section II, in spite of the fact that the large-scale unburned mixture fingers are more pronounced in the former case. ${ }^{65,71}$ Furthermore, a laminar premixed flame can be hydrodynamically unstable only if the perturbation length scale is larger than the so-called neutral length scale, 

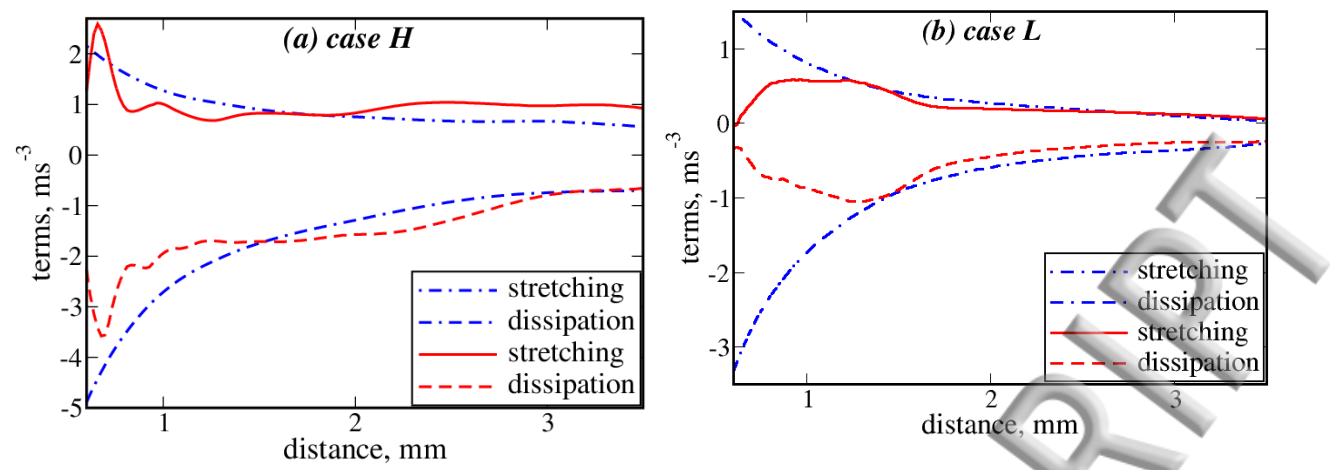

FIG. 18. Vortex stretching (dotted-dashed and solid lines) and dissipation (double-dashed-dotted and dashed lines) terms conditioned to unburned gas $(c(\boldsymbol{x}, t) \leq 0.001$, blue lines $)$ and cold edges of flamelets $(0.001<$ $c(\boldsymbol{x}, t) \leq 0.01$, red lines) vs. distance from the inlet boundary. (a) case $\mathrm{H}$, (b) case L.

which is much larger than the flame thickness. ${ }^{38,39}$ Accordingly, the DL instability is a large-scale phenomenon, whereas vorticity $\boldsymbol{\omega} \equiv \nabla \times \boldsymbol{u}$ characterizes the small-scale spatial variability of the velocity field by definition.

As far as the vorticity field in the incoming constant-density turbulent flow of the unburned reactants, the present authors are not aware of any investigation of the influence of the aforementioned pressure and potential velocity perturbations on the $\boldsymbol{\omega}$-field. Accordingly, to gain insight into such eventual effects, each term in Eq. (3) was averaged (over transverse plane and time) and conditioned either to the unburned reactants, i.e. $c(x, t) \leq 0.001$, or to the cold edges of flamelets, i.e., $0.001<c(\boldsymbol{x}, t) \leq 0.01$. As expected, the magnitudes of the conditioned dilatation and baroclinic torque terms were negligible when compared to the magnitudes of (i) the vortex-stretching terms $\bar{T}_{1, u}$ and $\bar{T}_{1, f}$ conditioned to $c(\boldsymbol{x}, t) \leq 0.01$ and $0.001<c(\boldsymbol{x}, t) \leq 0.01$, respectively, or (ii) the conditioned dilatation terms $\bar{T}_{2, u}$ and $\bar{T}_{2, f}$, respectively. Therefore, solely the conditioned vortexstretching terms $\bar{T}_{1, u}[\bar{c}(x)]$ and $\bar{T}_{1, f}[\bar{c}(x)]$ (dotted-dashed and solid lines, respectively) and the conditioned dissipation terms $\bar{T}_{2, u}[\bar{c}(x)]$ and $\bar{T}_{2, f}[\bar{c}(x)]$ (double-dashed-dotted and dashed lines, respectively) are plotted in Fig. 18.

These results show a minor difference between terms $\bar{T}_{1, u}[\bar{c}(x)]$ and $\bar{T}_{1, f}[\bar{c}(x)]$ or between $\bar{T}_{2, u}[\bar{c}(x)]$ and $\bar{T}_{2, f}[\bar{c}(x)]$ in the largest part of the mean flame brushes, i.e., at $x>1.5 \mathrm{~mm}$ or at $\bar{c}(x)>0.25$ in case $\mathrm{H}$ and $\bar{c}(x)>0.1$ in case L. However, at smaller $\bar{c}(x)$, the magnitudes of the terms conditioned to the cold edges of flamelets are lower than the magnitudes of the terms conditioned to the unburned reactants. For instance, at $x=0.66 \mathrm{~mm}$ or $\bar{c}(x)=6 \cdot 10^{-5}$ in case $\mathrm{H},\left|\bar{T}_{1, f}+\bar{T}_{2, f}\right|=0.83 \mathrm{~ms}^{-3}$, whereas $\left|\bar{T}_{1, u}+\bar{T}_{2, u}\right|=2.45 \mathrm{~ms}^{-3}$ is larger by a factor of about three. Such results imply that combustion-induced potential perturbations of the velocity field in the unburned gas in the vicinity of the cold edges of flamelets can locally slow down the spatial decay of vorticity in these regions (the turbulence decays in the $x$-direction in the present DNS and $\bar{T}_{1, u}+\bar{T}_{2, u}<0$ ), but further research into the issue is definitely required using a larger set of DNS data.

For this purpose, at each instant $t$, the vortex-stretching and dilatation terms, $\hat{T}_{1, u}(t)$ and $\hat{T}_{2, u}(t)$, respectively, were averaged over the leading zone of the mean flame brush, i.e., over a volume bounded by planes $\langle c\rangle(x, t)=10^{-6}$ and $\langle c\rangle(x, t)=10^{-4}$, whereas their conditioned counterparts $\hat{T}_{1, f}(t)$ and $\hat{T}_{2, f}(t)$, respectively, were averaged over a sub-volume where not only $10^{-6} \leq\langle c\rangle(x, t) \leq$ $10^{-4}$, but also $0.001<c(x, t) \leq 0.01$. Here, $\langle q\rangle(x, t)$ and $\hat{q}(t)$ designate values of an arbitrary quantity $q$, averaged over a transverse plane $x=$ const and a volume bounded by two transverse planes, respectively, at a single instant $t$.

The obtained results are reported in Fig. 19. A comparison of $\hat{T}_{1, u}(t)$ and $\hat{T}_{1, f}(t)$ (dotted-dashed and solid lines) or $\hat{T}_{2, u}(t)$ and $\hat{T}_{2, f}(t)$ (double-dashed-dotted and dashed lines) shows a decrease in the magnitudes of both terms conditioned to the flamelet cold edges when compared to the counter- 

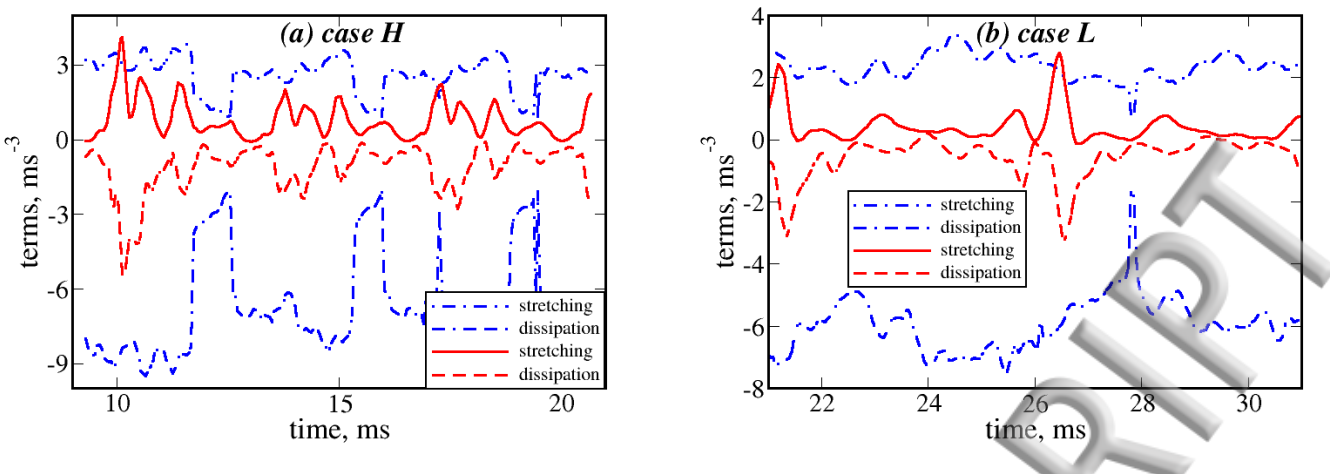

FIG. 19. Vortex stretching (dotted-dashed and solid lines) and dissipation (double-dashed-dotted and dashed lines) terms averaged over the leading zone (blue lines) of the mean flame brush and cold edges of flamelets $(0.001<c(\boldsymbol{x}, t) \leq 0.01$, red lines) within the leading zone at various instants. (a) case H, (b) case L.

part terms averaged over the entire volume of the leading zone of the flame brush. Accordingly, the net rate of the decay of enstrophy is reduced in the vicinity of the flamelet cold edges. However, a correlation of the effect magnitude with the density ratio is not observed.

\section{. IV. CONCLUSIONS}

DNS data analyzed in the present paper indicate that vorticity generated by baroclinic torque in a weakly turbulent premixed flame can impede increasing the reaction-zone-surface area, contrary to the common concept of combustion acceleration due to flame-generated turbulence. Such a smallscale effect is more pronounced at larger values of the mean combustion progress variable $\bar{c}$ and at larger density ratios. If the density ratio is low, e.g., $\sigma=2.5$, baroclinic torque weakly affects the vorticity field within the mean flame brush and the aforementioned effect is not pronounced.

It is worth stressing that the present work does not aim at claiming that the influence of combustion-induced thermal expansion on turbulence reduces the reaction-surface area and, hence, the burning rate. The potential velocity perturbations of larger scales can overwhelm the smallscale rotational perturbations and can result in increasing the burning rate, as occurs in the case of a hydrodynamically unstable laminar premixed flame ${ }^{37}$ For instance, large-scale unburned mixture fingers discussed in detail elsewhere ${ }^{65,71}$ imply an increase in flame surface area and turbulent burning rate due to combustion-induced thermal expansion.

However, if turbulence is considered to be the inherently rotational flow, then, the influence of the flame-generated turbulence on the burning rate appears to be fundamentally different from the influence of the turbulence in the incoming reactants on the rate. Accordingly, the concept of combustion acceleration due to flame-generated turbulence should be revisited and models developed for predicting an increase in the burning rate by the vorticity in the incoming turbulent flow are unlikely to be useful for describing a decrease in the reaction-surface area and, hence, the burning rate due to the flame-generated vorticity. In other words, the influence of the incoming turbulence on the burning rate should clearly be distinguished from the influence of the flame-generated vorticity on the rate and the two effects can be opposite.

\section{ACKNOWLEDGMENTS}

ANL gratefully acknowledges the financial support by the Combustion Engine Research Center. VAS gratefully acknowledges the financial support by ONERA and by the Grant of the Ministry of Education and Science of the Russian Federation (Contract No. 14.G39.31.0001 of 13.02.2017). 
This manuscript was accepted by Phys. Fluids. Click here to see the version of record.

${ }^{1}$ P. E. Hamlington, A. Y. Poludnenko, and E. S. Oran, "Interactions between turbulence and flames in premixed reacting flows," Phys. Fluids 23, 125111 (2011).

${ }^{2}$ N. Chakraborty, I. Konstantinou, and A. N. Lipatnikov, "Effects of Lewis number on vorticity and enstrophy transport in turbulent premixed flames," Phys. Fluids 28, 015109 (2016).

${ }^{3}$ B. Coriton and J. H. Frank, "Experimental study of vorticity-strain rate interaction in turbulent partially premixed jet flames using tomographic particle image velocimetry," Phys. Fluids 28, 025109 (2016).

${ }^{4}$ H. Wang, E. R. Hawkes, and J. H. Chen, "Turbulence-flame interactions in DNS of a laboratory high Karlovitz premixed turbulent jet flame," Phys. Fluids 28, 095107 (2016).

${ }^{5}$ C. Dopazo, L. Cifuentes, and N. Chakraborty, "Vorticity budgets in premixed combusting turbulent flows at different Lewis numbers," Phys. Fluids 29, 045106 (2017).

${ }^{6} \mathrm{Z}$. Wang and J. Abraham, "Effects of Karlovitz number on turbulent kinetic energy transport in turbulent lean premixed methane/air flames," Phys. Fluids 29, 085102 (2017).

${ }^{7}$ G. V. Nivarti and R. S. Cant, "Scalar transport and the validity of Damköhler's hypotheses for flame propagation in intense turbulence," Phys. Fluids 29, 085107 (2017).

${ }^{8}$ L. Cifuentes, C. Dopazo, A. Sandeep, N. Chakraborty, and A. Kempf, "Analysis of flame curvature evolution in a turbulent premixed bluff body burner," Phys. Fluids 30, 095101 (2018).

9 A. Jain and S. H. Kim, "On the non-equilibrium models for subfilter scalar variance in large eddy simulation of turbulent mixing and combustion," Phys. Fluids 31, 025112 (2019).

${ }^{10}$ S. Chaudhuri, V. Akkerman, and C. K. Law, "Accelerative propagation and explosion triggering by expanding turbulent premixed flames," Phys. Rev. E 87, 023008 (2013).

${ }^{11}$ V. N. Gamezo, A. M. Khokhlov, E. S. Oran, A. Y. Chtchelkanova, and R. O. Rosenberg, "Thermonuclear supernovae: simulations of the deflagration stage and their implications," Science 299, 77 (2003).

${ }^{12}$ V. N. Gamezo, A. M. Khokhlov, and E. S. Oran, "Deflagrations and detonations in thermonuclear supernovae," Phys. Rev. Lett. 92, 211102 (2004).

${ }^{13}$ K. N. C. Bray, "Turbulent flows with premixed reactants," in Turbulent Reacting Flows, edited by P. A. Libby and F. A. Williams (Springer-Verlag, Berlin, Germany, 1980), p. 115-183.

${ }^{14}$ F. A. Williams, Combustion Theory, 2nd ed. (Benjamin/Cummings, Menlo Park, California, 1985).

${ }^{15}$ R. Borghi, “Turbulent combustion modeling,” Prog. Energy Combust. Sci. 14, 245 (1988).

${ }^{16}$ V. R. Kuznetsov and V. A. Sabelnikov, Turbulence and Combustion (Hemisphere Publ. Corp., New York, 1990).

${ }^{17}$ J. Chomiak, Combustion: a Study in Theory, Fact and Application (Gordon and Breach, New York, 1990).

${ }^{18}$ N. Peters, Turbulent Combustion (Cambridge University Press, Cambridge, UK, 2000).

${ }^{19}$ A. N. Lipatnikov, Fundamentals of Premixed Turbulent Combustion (CRC Press, Boca Raton, Florida, 2012).

${ }^{20}$ B. Karlovitz, D. W. Denniston, and F. E. Wells, "Investigation of turbulent flames," J. Chem. Phys. 19, 541 (1951).

${ }^{21}$ A. C. Scurlock and J. H. Grover, "Propagation of turbulent flames," Symp. (Int.) Combust. 4, 645 (1953).

${ }^{22}$ R. Günther, "Turbulence properties of flames and their measurement," Prog. Energy Combust. Sci. 9, 105 (1983).

${ }^{23}$ K. N. C. Bray, “Turbulent transport in flames," Proc. R. Soc. London A 451, 231 (1995).

${ }^{24}$ A. N. Lipatnikov and J. Chomiak, "Effects of premixed flames on turbulence and turbulent scalar transport," Prog. Energy Combust. Sci. 36, 1 (2010).

${ }^{25}$ V. A. Sabelnikoy and A. N. Lipatnikov, "Recent advances in understanding of thermal expansion effects in premixed turbulent flames," Annu. Rev. Fluid Mech. 49, 91 (2017).

${ }^{26}$ A. N. Lipatnikov and J. Chomiak, "Turbulent flame speed and thickness: phenomenology, evaluation, and application in multi-dimensional simulations," Prog. Energy Combust. Sci. 28, 1 (2002).

${ }^{27}$ Y. A. Gostintsev, A. G. Istratov, and Y. V. Shulenin, "Self-similar propagation of a free turbulent flame in mixed gas mixtures," Combust. Explosion Shock Waves 24, 563 (1988).

${ }^{28}$ Y. A. Gostintsev, A. G. Istratov, N. I. Kidin, and V. E. Fortov, "Self-turbulization of gas flames: An analysis of experimental results," High Temperature 37(2), 282 (1999).

${ }^{29}$ D. Bradley, "Instabilities and flame speeds in large-scale premixed gaseous explosions," Phil. Trans. R. Soc. London 357, 3567 (1999).

${ }^{30}$ R. Addabbo, J. K. Bechtold, and M. Matalon, "Wrinkling of spherically expanding flames," Proc. Combust. Inst. 29, 1527 (2002).

${ }^{31}$ G. I. Sivashinsky, "Some developments in premixed combustion modeling," Proc. Combust. Inst. 29, 1737 (2002).

${ }^{32}$ M. Matalon, "Flame dynamics," Proc. Combust. Inst. 32, 57 (2009).

${ }^{33}$ V. Akkerman, C. K. Law, and V. Bychkov, "Self-similar accelerative propagation of expanding wrinkled flames and explosion triggering," Phys. Rev. E 83, 026305 (2011).

${ }^{34}$ A. R. Kerstein, “Turbulence in combustion processes: modeling challenges,” Proc. Combust. Inst. 29, 1763 (2002).

${ }^{35}$ A. Tsinober, An Informal Conceptual Introduction to Turbulence (Springer, Berlin, Germany, 2009). 
${ }^{36}$ A. N. Lipatnikov, V. A. Sabelnikov, S. Nishiki, and T. Hasegawa, "Does flame-generated vorticity increase turbulent burning velocity?" Phys. Fluids 30, 081702 (2018).

${ }^{37}$ L. D. Landau and E. M. Lifshitz, Fluid Mechanics (Pergamon Press, Oxford, UK, 1987).

${ }^{38}$ P. Clavin, "Dynamical behavior of premixed flame fronts in laminar and turbulent flows," Prog. Energy Combust. Sci. 11, 1 (1985).

${ }^{39}$ M. Matalon, "Intrinsic flame instabilities in premixed and nonpremixed combustion," Annu. Rev. Fluid Mech. 39, 163 (2007).

${ }^{40}$ M. L. Frankel, "An equation of surface dynamics modeling flame fronts as density discontinuities in potential flows," Phys. Fluids A 2, 1879 (1990).

${ }^{41}$ G. I. Sivashinsky and P. Clavin, "On the nonlinear theory of hydrodynamic instability in flames," J. Physique 48, 193 (1987).

${ }^{42}$ A. N. Lipatnikov, S. Nishiki, and T. Hasegawa, "A direct numerical simulation study of vorticity transformation in weakly turbulent premixed flames," Phys. Fluids 26, 105104 (2014).

${ }^{43}$ B. Bobbitt, S. Lapointe, and G. Blanquart, "Vorticity transformation in high Karlovitz number premixed flames," Phys. Fluids 28, 015101 (2016).

44 S. H. R. Whitman, C. A. Z. Towery, A. Y. Poludnenko, and P. E. Hamlington, "Scaling and collapse of conditional velocity structure functions in turbulent premixed flames," Proc. Combust. Inst. 37, 2527 (2019).

${ }^{45}$ P. E. Hamlington, A. Y. Poludnenko, and E. S. Oran, "Intermittency in premixed turbulent reacting flows," Phys. Fluids 24, 075111 (2012).

${ }^{46}$ N. Chakraborty, L. Wang, I. Konstantinou, and M. Klein, "Vorticity statistics based on velocity and densityweighted velocity in premixed reactive turbulence," J. Turbulence 18, 825 (2017).

${ }^{47}$ S. Nishiki, T. Hasegawa, R. Borghi, and R. Himeno, "Modeling of flame-generated turbulence based on direct numerical simulation databases,” Proc. Combust. Inst. 29, 2017 (2002).

${ }^{48}$ S. Nishiki, T. Hasegawa, R. Borghi, and R. Himeno, "Modelling of turbulent scalar flux in turbulent premixed flames based on DNS databases," Combust. Theory Modelling 10, 39 (2006).

${ }^{49}$ H. Carlsson, R. Yu, and X.-S. Bai, "Flame structure analysis for categorization of lean premixed $\mathrm{CH}_{4} /$ air and $\mathrm{H}_{2}$ /air flames at high Karlovitz numbers: Direct numerical simulation studies," Proc. Combust. Inst. 35, 1425 (2015).

${ }^{50}$ H. A. Uranakara, S. Chaudhuri, H. L. Dave, P. G. Arias, and H. G. Im, "A flame particle tracking analysis of turbulence-chemistry interaction in hydrogen-air premixed flames," Combust. Flame 163, 220 (2016).

${ }^{51}$ A. J. Aspden, M. J. Day, and J. B. Bell, "Three-dimensional direct numerical simulation of turbulent lean premixed methane combustion with detailed kinetics," Combust. Flame 165, 266 (2016).

${ }^{52}$ D. Cecere, E. Giacomazzi, N. M. Arcidiacono, and F. R. Picchia, "Direct numerical simulation of a turbulent lean premixed $\mathrm{CH}_{4} / \mathrm{H}_{2}$-air slot flame," Combust. Flame 165, 384 (2016).

${ }^{53}$ H. Wang, E. R. Hawkes, B. Zhou, J. H. Chen, Z. Li, and M. Aldén, "A comparison between direct numerical simulation and experiment of the turbulent burning velocity-related statistics in a turbulent methane-air premixed jet flame at high Karlovitz number," Proc. Combust. Inst. 36, 2045 (2017).

${ }^{54}$ S. Chaudhuri, H. Kolla, H. L. Dave, E. R. Hawkes, J. H. Chen, and C.K. Law, "Flame thickness and conditional scalar dissipation rate in a premixed temporal turbulent reacting jet," Combust. Flame 184, 273 (2017).

${ }^{55}$ J. F. MacArt, T. Grenga, and M. E. Mueller, "Effects of combustion heat release on velocity and scalar statistics in turbulent premixed jet flames at low and high Karlovitz numbers," Combust. Flame 191, 468 (2018).

${ }^{56}$ Y. Minamoto, B. Yenerdag, and M. Tanahashi, "Morphology and structure of hydrogen-air turbulent premixed flames," Combust. Flame 192, 369 (2018).

${ }^{57}$ D. Veynante and L. Vervisch, "Turbulent combustion modeling," Prog. Energy Combust. Sci. 28, 193 (2002).

${ }^{58}$ A. N. Lipatnikov, S. Nishiki, and T. Hasegawa, "DNS assessment of relation between mean reaction and scalar dissipation rates in the flamelet regime of premixed turbulent combustion," Combust. Theory Modell. 19, 309 (2015)

${ }^{59}$ Y. H. Im, K. Y. Huh, S. Nishiki, and T. Hasegawa, "Zone conditional assessment of flame-generated turbulence with DNS database of a turbulent premixed flame," Combust. Flame 137, 478 (2004).

${ }^{60}$ A. Mura, K. Tsuboi, and T. Hasegawa, "Modelling of the correlation between velocity and reactive scalar gradients in turbulent premixed flames based on DNS data," Combust. Theory Modelling 12, 671 (2008).

${ }^{61}$ A. Mura, V. Robin, M. Champion, and T. Hasegawa, "Small scale features of velocity and scalar fields in turbulent premixed flames," Flow Turbul. Combust. 82, 339 (2009).

${ }^{62}$ V. Robin, A. Mura, M. Champion, and T. Hasegawa, "Direct and indirect thermal expansion effects in turbulent premixed flames," Combust. Sci. Technol. 182, 449 (2010).

${ }^{63}$ V. Robin, A. Mura, and M. Champion, "Modeling of the effects of thermal expansion on scalar turbulent fluxes in turbulent premixed flames," J. Fluid Mech. 689, 149 (2011).

${ }^{64}$ K. N. C. Bray, M. Champion, P. A. Libby, and N. Swaminathan, "Scalar dissipation and mean reaction rates in premixed turbulent combustion,” Combust. Flame 158, 2017 (2011). 
${ }^{65}$ A. N. Lipatnikov, J. Chomiak, V. A. Sabelnikov, S. Nishiki, and T. Hasegawa, "Unburned mixture fingers in premixed turbulent flames," Proc. Combust. Inst. 35, 1401 (2015).

${ }^{66}$ A. N. Lipatnikov, V. A. Sabelnikov, S. Nishiki, T. Hasegawa, and N. Chakraborty, "DNS assessment of a simple model for evaluating velocity conditioned to unburned gas in premixed turbulent flames," Flow Turbul. Combust. 94, 513 (2015).

${ }^{67}$ V. A. Sabelnikov, A. N. Lipatnikov, N. Chakraborty, S. Nishiki, and T. Hasegawa, "A transport equation for reaction rate in turbulent flows," Phys. Fluids 28, 081701 (2016).

${ }^{68}$ V. A. Sabelnikov, A. N. Lipatnikov, N. Chakraborty, S. Nishiki, and T. Hasegawa, "A balance equation for the mean rate of product creation in premixed turbulent flames," Proc. Combust. Inst. 36, 1893 (2017).

${ }^{69}$ A. N. Lipatnikov, V. A. Sabelnikov, S. Nishiki, and T. Hasegawa, "Flamelet perturbations and flame surface density transport in weakly turbulent premixed combustion," Combust. Theory Modell. 21, 205 (2017).

${ }^{70}$ A. N. Lipatnikov, V. A. Sabelnikov, N. Chakraborty, S. Nishiki, and T. Hasegawa, "A DNS study of closure relations for convection flux term in transport equation for mean reaction rate in turbulent flow," Flow Turbul. Combust. 100, 75 (2018).

${ }^{71}$ A. N. Lipatnikov, J. Chomiak, V. A. Sabelnikov, S. Nishiki, and T. Hasegawa, "A DNS study of the physical mechanisms associated with density ratio influence on turbulent burning velocity in premixed flames," Combust. Theory Modell. 22, 131 (2018).

${ }^{72}$ A. N. Lipatnikov, V. A. Sabelnikov, S. Nishiki, and T. Hasegawa, "Combustion-induced local shear layers within premixed flamelets in weakly turbulent flows," Phys. Fluids 30, 085101 (2018).

${ }^{73}$ V. A. Sabelnikov, A. N. Lipatnikov, S. Nishiki, and T. Hasegawa, "Application of conditioned structure functions to exploring influence of premixed combustion on two-point turbulence statistics," Proc. Combust. Inst. 37, 2433 (2019).

${ }^{74}$ A. N. Lipatnikov, S. Nishiki, and T. Hasegawa, "A DNS assessment of linear relations between filtered reaction rate, flame surface density, and scalar dissipation rate in a weakly turbulent premixed flame," Combust. Theory Modell. 23, in press (2019).

${ }^{75}$ V. A. Sabelnikov, A. N. Lipatnikov, S. Nishiki, and T. Hasegawa, "Investigation of the influence of combustion-induced thermal expansion on two-point turbulence statistics using conditioned structure functions," J. Fluid Mech. 867, 45 (2019).

${ }^{76}$ R. H. Kraichnan, "Decay of isotropic turbulence in the direct-interaction approximation," Phys. Fluids 7, 1030 (1964).

${ }^{77}$ Ya. B. Zel'dovich, G. I. Barenblatt, V. B. Librovich, and G. M. Makhviladze, The Mathematical Theory of Combustion and Explosions (Consultants Burea, New York, 1985).

${ }^{78}$ A. N. Lipatnikov and J. Chomiak, "Molecular transport effects on turbulent flame propagation and structure," Prog. Energy Combust. Sci. 31, 1 (2005).

${ }^{79}$ V. A. Sabelnikov and A. N. Lipatnikov, "Transition from pulled to pushed fronts in premixed turbulent combustion: theoretical and numerical study," Combust. Flame 162, 2893 (2015).

${ }^{80}$ A. N. Lipatnikov, N. Chakraborty, and V. A. Sabelnikov, "Transport equations for reaction rate in laminar and turbulent premixed flames characterized by non-unity Lewis number," Int. J. Hydrogen Energy 43, 21060 (2018).

${ }^{81}$ S. H. Kim, "Leading points and heat release effects in turbulent premixed flames," Proc. Combust. Inst. 36, 2017 (2017).

${ }^{82}$ H. L. Dave, A. Mohan, and S. Chaudhuri, "Genesis and evolution of premixed flames in turbulence," Combust. Flame 196, 386 (2018).

${ }^{83}$ P. Venkateswaran, A. Marshall, D. H. Shin, D. Noble, J. Seitzman, and T. Lieuwen, "Measurements and analysis of turbulent consumption speeds of $\mathrm{H}_{2} / \mathrm{CO}$ mixtures," Combust. Flame 158, 1602 (2011).

${ }^{84}$ P. Venkateswaran, A. Marshall, J. Seitzman, and T. Lieuwen, "Scaling turbulent flame speeds of negative Markstein length fuel blends using leading points concepts," Combust. Flame 162, 375 (2015).

${ }^{85}$ W. Zhang, J. Wang, Q. Yu, W. Jin, M. Zhang, and Z. Huang, "Investigation of the fuel effects on burning velocity and flame structure of turbulent premixed flames based on leading points concept," Combust. Sci. Technol. 190, 1354 (2018).

${ }^{86}$ M. Matalon, “On flame stretch,” Combust. Sci. Technol. 31, 169 (1983).

${ }^{87}$ S. B. Pope, "The evolution of surface in turbulence," Int. J. Engng Sci. 26, 445 (1988).

${ }^{88}$ S. Candel and T. Poinsot, "Flame stretch and the balance equation for the flame area," Combust. Sci. Technol. 170, 1(1990). 